\title{
Well-Posedness of Two-Dimensional Hydroelastic Waves
}

\author{
David M. Ambrose* Michael Siegel
}

November 11, 2014

\begin{abstract}
A well-posedness theory for the initial value problem for hydroelastic waves in two spatial dimensions is presented. This problem, which arises in numerous applications, describes the evolution of a thin elastic membrane in a two-dimensional potential flow. We use a model for the elastic sheet that accounts for bending stresses and membrane tension, but which neglects the mass of the membrane. The analysis is based on a vortex sheet formulation, and following earlier analyses and numerical computations in 2D interfacial flow with surface tension, we use an angle-arclength representation of the problem. We prove short-time well-posedness in Sobolev spaces. The proof is based on energy estimates, and the main challenge is to find a definition of the energy and estimates on high-order nonlocal terms so that an a priori bound can be obtained.
\end{abstract}

\section{Introduction}

The hydroelastic problem describes the interaction between elastic bodies and hydrodynamic flow. We are interested in the particular version of this problem where an elastic sheet or membrane evolves in a potential flow. This problem is important in biology, medicine, and ocean engineering, and arises, for example, as a model for the dynamics of flapping flags [1], heart valves [28], ice sheets in the ocean [36], and very large floating structures [35]. A review which summarizes recent work on the analysis, numerical simulation, and applications of the hydroelastic problem is given by Korobkin, Parau and Vanden Broeck [29].

This paper presents an existence and uniqueness theory for the initial value problem for hydroelastic waves. Recently, Plotnikov and Toland [32] derived nonlinear equations that model the interaction of a thin, heavy elastic sheet with a three-dimensional inviscid, irrotational fluid. Their derivation is based on the Cosserat theory of shells satisfying Kirchoff's hypothesis, and accounts for bending stresses in the sheet as well as a membrane stretching tension. A similar model for the bending stress can be derived from minimization of the Willmore energy functional. Here, we consider a model for the 2D hydroelastic time-evolution problem (that is, a $1 \mathrm{D}$ interface evolving in 2D fluid flow) that is consistent with the hydroelastic formulation of Plotnikov and Toland, but which neglects the mass of the elastic membrane. The model is derived follwing the approach of [12]. Our main result is the local well-posedness of this problem.

The mathematical analysis of fluid-structure interaction presents significant challenges, and the only rigourous results for the hydroelastic problem that we are aware of are on the existence of steady

*DMA gratefully acknowledges support from the National Science Foundation through grant DMS-1016267. 
traveling waves $[33,38,39]$. There is more work on the well-posedness of models for the interaction of a viscous (Navier-Stokes) fluid with an elastic membrane or solid. For example, Cheng et al. [14] have developed an existence and uniqueness theory for the problem of a nonlinear elastic 'bio-fluid' shell interacting with a viscous incompressible bulk fluid governed by the Navier-Stokes equations. Their model for the elastic shell involves a bending stress which extremizes the Willmore energy functional, similar to here, and a membrane or surface energy that is a function of the local area ratio. Wang et al. [40] prove the local well-posedness of an elastic surface model consisting of a viscous incompressible membrane fluid, but neglect the interaction with the bulk fluid. Their work generalizes an earlier analysis by $\mathrm{Hu}$ et al. [27]. Other work includes existence results for the interaction of a viscous fluid with, respectively, an elastic body moving in the fluid interior (e.g. [20]), finite thickness elastic shells [15], and regularized models of elastic plates (e.g. [13]). At first glance, one might surmise that theory developed in this paper can follow from the zero viscosity limit of [14]. A difficulty with this idea is that the Navier-Stokes and Euler's equations admit different kinds of boundary conditions, and the relationship between the zero viscosity limit of Navier-Stokes and Euler remains an open problem.

The approach developed in this paper relies on a boundary integral formulation of the hydroelastic problem. The analysis uses several important ideas from the numerical work of Hou, Lowengrub and Shelley (HLS) [25] and the analysis of Ambrose [3] of the initial value problem for vortex sheets with surface tension. These works recast the evolution equation by using the tangent angle $\theta$ and the arclength $s$ as the dependent variables, rather than the natural Cartesian variables $x$ and $y$. This choice of variables simplifies the curvature terms in the evolution equations. Additionally, they make a special choice of the tangential velocity $V(\alpha, t)$ of the interface (which may be chosen arbitrarily, and defines the parameterization $\alpha$ ) so that $s_{\alpha}$ is independent of $\alpha$. With this choice of $V$, the parameterization, normalized so that $\alpha$ is between 0 and $2 \pi$, is an equal arclength parameterization. There is a jump in velocity at the interface which is then a vortex sheet, and we denote the vortex sheet strength by $\gamma$. These choices simplify the analysis since the leading order or high derivative terms are linear as functions of $\theta$ and $\gamma$.

The analysis requires special care in the handling of terms with the highest number of spatial derivatives, which are contained within a singular integral operator known as the Birkhoff-Rott integral. To facilitate this, our proof makes use of the small scale decomposition (SSD) introduced in HLS. In the SSD, the leading order or highest derivative terms that are dominant at small spatial scales are identified and written in a simple form involving Hilbert transforms, rather than the more complicated Birkhoff-Rott integral. The small scale decomposition was used in HLS for computational purposes, but here it is employed in an essential way to simplify terms that need to be treated carefully in the analysis.

The main result of this paper is that the initial value problem for the hydroelastic flow of a periodic interface is well-posed in Sobolev spaces. In particular, given periodic initial data $\theta(\cdot,) \in H^{s}$ and $\gamma(\cdot, 0) \in H^{s-3 / 2}$ for $s$ large enough (so that the interface variables $x(\alpha, t)$ and $y(\alpha, t)$ are in $H^{s+1}$ ) there is a nonzero time in which the solution exists, is unique, has the same regularity as the initial conditions, and depends continuously on the data.

The proof uses energy methods. The analysis is similar to that for vortex sheets with surface tension in [3], and the main challenge compared to the previous analysis is that the elasticity introduces higher order (nonlocal) terms, some of which are nonlinear. This requires a different definition of energy and more care in the energy estimates to achieve closure, that is, a bound on the time derivative of the energy by a function of the energy itself, which is a critical step in the proof.

The rest of this paper is organized as follows. In $\S 2$ we present an instructive example that illus- 
trates the essential features of the energy estimate. Governing equations and preliminary estimates are presented in $\S 3$ and $\S 4$. The main existence proof is given in $\S 5$, and uniqueness and continuous dependence of the solution on the data is demonstrated in $\S 6$. Concluding remarks are given in $\S 7$. The appendix $\S 8$ derives an expression for the pressure jump at the interface that is used in the model.

\subsection{Function spaces, norms, operators, and notation}

Derivatives with respect to the independent variables $t$ and $\alpha$ will be denoted either by using the partial derivative operators $\partial_{t}$ and $\partial_{\alpha}$, or with subscripts; thus $f_{t}=\partial_{t} f, f_{\alpha}=\partial_{\alpha} f, f_{\alpha \alpha}=\partial_{\alpha}^{2} f$, and so on.

We comment now about the function spaces we will use. We use the $L^{2}$-based Sobolev spaces in the $2 \pi$-periodic setting. For $f \in H^{k}$ with $k \in \mathbb{N}$, we use the following as the norm:

$$
\|f\|_{k}=\left(\int_{0}^{2 \pi} f^{2}(\alpha)+\left(\partial_{\alpha}^{k} f(\alpha)\right)^{2} d \alpha\right)^{1 / 2} .
$$

For $f \in H^{k+\frac{1}{2}}$, with $k \in \mathbb{N}$, we use the following as the norm:

$$
\|f\|_{k+\frac{1}{2}}=\left(\int_{0}^{2 \pi} f^{2}(\alpha)+\left(\partial_{\alpha}^{k} f(\alpha)\right)\left(H \partial_{\alpha}^{k+1} f(\alpha)\right) d \alpha\right)^{1 / 2} .
$$

Here, $H$ is the periodic Hilbert transform, which has symbol $\hat{H}(\xi)=-i \operatorname{sgn}(\xi)$. (Notice that if $f$ has mean zero, then $H^{2} f=-f$. For more information on the periodic Hilbert transform, the interested reader might consult [24]). Using Plancherel's Theorem, it is clear that

$$
\|f\|_{k+\frac{1}{2}}=\left(\sum_{\xi}\left(1+|\xi|^{2 k+1}\right)|\hat{f}(\xi)|^{2}\right)^{1 / 2}
$$

so this is equivalent to any other usual definition of the $H^{k+\frac{1}{2}}$ norm.

We will frequently use the notation $\Lambda$ for the operator $\Lambda=H \partial_{\alpha}$; with this definition, the symbol of $\Lambda$ is $\hat{\Lambda}(\xi)=|\xi|$, and this implies that $\Lambda$ is self-adjoint. This implies the following, which we will use many times:

$$
\frac{d}{d t} \int_{0}^{2 \pi} g \Lambda g d \alpha=2 \int_{0}^{2 \pi} g \Lambda g_{t} d \alpha=2 \int_{0}^{2 \pi} g_{t} \Lambda g d \alpha .
$$

This will be relevant as we estimate the growth of quantities which are equivalent to $H^{k+\frac{1}{2}}$ norms.

We will sometimes use the projection $\mathbb{P}$, which removes the zero mode of a periodic function:

$$
\mathbb{P} f=f-\frac{1}{2 \pi} \int_{0}^{2 \pi} f(\alpha) d \alpha
$$

We may sometimes denote the mean of a periodic function as $\langle\langle f\rangle$, so that we could say $\mathbb{P} f=f-\langle\langle f\rangle$. We also introduce the mean-zero antiderivative operator, $\partial_{\alpha}^{-1}$. This is defined through its symbol as $\widehat{\partial_{\alpha}^{-1} f}(k)=\frac{1}{i k} \hat{f}(k)$, for $k \neq 0$, and $\widehat{\partial_{\alpha}^{-1} f}(0)=0$. 


\section{An instructive example}

Let $c_{1}, c_{2}, c_{3}, c_{4}$, and $c_{5}$ all be positive constants. We consider the following linear system, which has the same types of leading-order terms as the hydroelastic wave system we will be studying.

$$
\begin{gathered}
\theta_{t}=H\left(\gamma_{\alpha}\right)+\left(c_{1}-c_{2}\right) \partial_{\alpha}^{-1} H(\gamma), \\
\gamma_{t}=-c_{3} \theta_{\alpha \alpha \alpha \alpha}+\left(c_{4}-c_{5}\right) \theta_{\alpha \alpha} .
\end{gathered}
$$

The coefficients of the second terms on the right-hand sides, which are $c_{1}-c_{2}$ and $c_{4}-c_{5}$, are written this way to make clear that we will be able to estimate these terms regardless of whether these coefficients are positive or negative. For the purpose of the present example, we take $(\theta, \gamma)$ to be a solution of this system which is sufficiently smooth for all of the integrals we are about to use to make sense.

The energy we will estimate will serve as an upper bound for a constant times the square of the $H^{3}$-norm of $\theta$ and the square of the $H^{3 / 2}$-norm of $\gamma$. We let $E(t)$ be given by

$$
E(t)=E_{0}(t)+E_{1}(t)+E_{2}(t)+E_{3}(t)+E_{4}(t),
$$

where

$$
\begin{gathered}
E_{0}(t)=\frac{1}{2} \int_{0}^{2 \pi} \theta^{2}+\gamma^{2} d \alpha, \\
E_{1}(t)=\frac{c_{3}}{2} \int_{0}^{2 \pi}\left(\partial_{\alpha}^{3} \theta\right)^{2} d \alpha, \\
E_{2}(t)=\frac{1}{2} \int_{0}^{2 \pi}\left(\partial_{\alpha} \gamma\right)\left(\Lambda \partial_{\alpha} \gamma\right) d \alpha,
\end{gathered}
$$

and $E_{3}$ and $E_{4}$ will be defined shortly.

Taking the time derivative, it is straightforward that the growth of $E_{0}$ is bounded in terms of $E$ :

$$
\frac{d E_{0}}{d t} \leq c E
$$

We next take the time derivative of $E_{1}$ :

$$
\frac{d E_{1}}{d t}=\int_{0}^{2 \pi}\left(\partial_{\alpha}^{3} \theta\right)\left(\partial_{\alpha}^{3} \theta_{t}\right) d \alpha
$$

Substituting from the evolution equation for $\theta$, this is

$$
\frac{d E_{1}}{d t}=c_{3} \int_{0}^{2 \pi}\left(\partial_{\alpha}^{3} \theta\right)\left(H \partial_{\alpha}^{4} \gamma\right) d \alpha+c_{3}\left(c_{1}-c_{2}\right) \int_{0}^{2 \pi}\left(\partial_{\alpha}^{3} \theta\right)\left(H \partial_{\alpha}^{2} \gamma\right) d \alpha
$$

Next, we take the time derivative of $E_{2}$ :

$$
\frac{d E_{2}}{d t}=\int_{0}^{2 \pi}\left(\partial_{\alpha} \gamma\right)\left(\Lambda \partial_{\alpha} \gamma_{t}\right) d \alpha
$$

Plugging in from the evolution equation, this is

$$
\frac{d E_{2}}{d t}=-c_{3} \int_{0}^{2 \pi}\left(\partial_{\alpha} \gamma\right)\left(\Lambda \partial_{\alpha}^{5} \theta\right) d \alpha+\left(c_{4}-c_{5}\right) \int_{0}^{2 \pi}\left(\partial_{\alpha} \gamma\right)\left(\Lambda \partial_{\alpha}^{3} \theta\right) d \alpha
$$


We use the fact that $\Lambda$ is self-adjoint, and we use the definition $\Lambda=H \partial_{\alpha}$ :

$$
\frac{d E_{2}}{d t}=-c_{3} \int_{0}^{2 \pi}\left(H \partial_{\alpha}^{2} \gamma\right)\left(\partial_{\alpha}^{5} \theta\right) d \alpha+\left(c_{4}-c_{5}\right) \int_{0}^{2 \pi}\left(H \partial_{\alpha}^{2} \gamma\right)\left(\partial_{\alpha}^{3} \theta\right) d \alpha .
$$

We integrate by parts twice in the first integral on the right-hand side:

$$
\frac{d E_{2}}{d t}=-c_{3} \int_{0}^{2 \pi}\left(H \partial_{\alpha}^{4} \gamma\right)\left(\partial_{\alpha}^{3} \theta\right) d \alpha+\left(c_{4}-c_{5}\right) \int_{0}^{2 \pi}\left(H \partial_{\alpha}^{2} \gamma\right)\left(\partial_{\alpha}^{3} \theta\right) d \alpha .
$$

We add (3) and (4), finding that the terms with the most derivatives cancel. We are left with

$$
\frac{d E_{1}}{d t}+\frac{d E_{2}}{d t}=\left[\left(c_{4}+c_{1} c_{3}\right)-\left(c_{5}+c_{2} c_{3}\right)\right] \int_{0}^{2 \pi}\left(H \partial_{\alpha}^{2} \gamma\right)\left(\partial_{\alpha}^{3} \theta\right) d \alpha .
$$

Notice that this integral is not bounded in terms of the energy, since the energy controls three derivatives of $\theta$ and $3 / 2$ of a derivative of $\gamma$; thus, this integral contains terms with $1 / 2$ of a derivative more than we can control. We will cancel this by using $E_{3}$ and $E_{4}$, which we now define as

$$
\begin{aligned}
& E_{3}(t)=\frac{d_{1}}{2} \int_{0}^{2 \pi}\left(\partial_{\alpha}^{2} \theta\right)^{2} d \alpha, \\
& E_{4}(t)=\frac{d_{2}}{2} \int_{0}^{2 \pi} \gamma(\Lambda \gamma) d \alpha .
\end{aligned}
$$

The positive constants $d_{1}$ and $d_{2}$ will be specified soon.

We take the time derivative of $E_{3}$ :

$$
\frac{d E_{3}}{d t}=d_{1} \int_{0}^{2 \pi}\left(\partial_{\alpha}^{2} \theta\right)\left(\partial_{\alpha}^{2} \theta_{t}\right) d \alpha
$$

Plugging in from the evolution equation, this is

$$
\frac{d E_{3}}{d t}=d_{1} \int_{0}^{2 \pi}\left(\partial_{\alpha}^{2} \theta\right)\left(H \partial_{\alpha}^{3} \gamma\right) d \alpha+d_{1}\left(c_{1}-c_{2}\right) \int_{0}^{2 \pi}\left(\partial_{\alpha}^{2} \theta\right)\left(H \partial_{\alpha} \gamma\right) d \alpha
$$

We integrate by parts once in the first integral on the right-hand side:

$$
\frac{d E_{3}}{d t}=-d_{1} \int_{0}^{2 \pi}\left(\partial_{\alpha}^{3} \theta\right)\left(H \partial_{\alpha}^{2} \gamma\right) d \alpha+d_{1}\left(c_{1}-c_{2}\right) \int_{0}^{2 \pi}\left(\partial_{\alpha}^{2} \theta\right)\left(H \partial_{\alpha} \gamma\right) d \alpha .
$$

We next take the time derivative of $E_{4}$ :

$$
\frac{d E_{4}}{d t}=d_{2} \int_{0}^{2 \pi} \gamma\left(\Lambda \gamma_{t}\right) d \alpha .
$$

We plug in from the evolution equation, finding the following:

$$
\frac{d E_{4}}{d t}=-c_{3} d_{2} \int_{0}^{2 \pi} \gamma\left(\Lambda \partial_{\alpha}^{4} \theta\right) d \alpha+d_{2}\left(c_{4}-c_{5}\right) \int_{0}^{2 \pi} \gamma\left(\Lambda \partial_{\alpha}^{2} \theta\right) d \alpha .
$$

For the first integral on the right-hand side, we use the fact that $\Lambda$ is self-adjoint, and we use the definition $\Lambda=H \partial_{\alpha}$, and we also integrate by parts once (we also use $\Lambda=H \partial_{\alpha}$ in the second integral):

$$
\frac{d E_{4}}{d t}=c_{3} d_{2} \int_{0}^{2 \pi}\left(H \partial_{\alpha}^{2} \gamma\right)\left(\partial_{\alpha}^{3} \theta\right) d \alpha+d_{2}\left(c_{4}-c_{5}\right) \int_{0}^{2 \pi} \gamma\left(H \partial_{\alpha}^{3} \theta\right) d \alpha .
$$


We now add (5), (6), and (7), to find the following:

$$
\begin{aligned}
\frac{d E_{1}}{d t}+\frac{d E_{2}}{d t}+\frac{d E_{3}}{d t}+\frac{d E_{4}}{d t} & =\left[-d_{1}+c_{3} d_{2}+c_{4}+c_{1} c_{3}-c_{5}-c_{2} c_{3}\right] \int_{0}^{2 \pi}\left(H \partial_{\alpha}^{2} \gamma\right)\left(\partial_{\alpha}^{3} \theta\right) d \alpha \\
& +d_{1}\left(c_{1}-c_{2}\right) \int_{0}^{2 \pi}\left(\partial_{\alpha}^{2} \theta\right)\left(H \partial_{\alpha} \gamma\right) d \alpha+d_{2}\left(c_{4}-c_{5}\right) \int_{0}^{2 \pi} \gamma\left(H \partial_{\alpha}^{3} \theta\right) d \alpha .
\end{aligned}
$$

If we choose

$$
d_{1}=c_{4}+c_{1} c_{3}, \quad d_{2}=\frac{c_{5}+c_{2} c_{3}}{c_{3}},
$$

then the first integral on the right-hand side of (8) vanishes. We then have

$$
\frac{d E_{1}}{d t}+\frac{d E_{2}}{d t}+\frac{d E_{3}}{d t}+\frac{d E_{4}}{d t}=d_{1}\left(c_{1}-c_{2}\right) \int_{0}^{2 \pi}\left(\partial_{\alpha}^{2} \theta\right)\left(H \partial_{\alpha} \gamma\right) d \alpha+d_{2}\left(c_{4}-c_{5}\right) \int_{0}^{2 \pi} \gamma\left(H \partial_{\alpha}^{3} \theta\right) d \alpha .
$$

Since the remaining integrals involve at most one derivative of $\gamma$ and at most three derivatives of $\theta$, this can be estimated in terms of the energy:

$$
\frac{d E_{1}}{d t}+\frac{d E_{2}}{d t}+\frac{d E_{3}}{d t}+\frac{d E_{4}}{d t} \leq c E
$$

Adding (2) and (9), we get

$$
\frac{d E}{d t}=\frac{d E_{0}}{d t}+\frac{d E_{1}}{d t}+\frac{d E_{2}}{d t}+\frac{d E_{3}}{d t}+\frac{d E_{4}}{d t} \leq c E .
$$

This implies that the energy grows at most exponentially. Since the constants $d_{1}, d_{2}$, and $c_{3}$ are positive, we have the following:

$$
\min \left\{\frac{1}{2}, \frac{c_{3}}{2}\right\}\left(\|\theta\|_{3}^{2}+\|\gamma\|_{3 / 2}^{2}\right) \leq E(t) \leq E(0) e^{c t} .
$$

Thus, the norm of $(\theta, \gamma)$ grows at most exponentially.

\section{Equations of motion}

In this section, we formulate the evolution equations for periodic hydroelastic waves, using the $\theta-L$ formulation of Hou, Lowengrub, and Shelley [25], [26]. This formulation has previously been used by the first author to develop the well-posedness theory of vortex sheets, water waves, and Hele-Shaw flows [3], [4], [5], [7]. Other authors have also used this formulation to prove results for water waves and Hele-Shaw flows including well-posedness, stability, and regularity results, among others [16], [17], [19], [21], [23], [42], [43].

We consider an interface $S$ separating two inviscid, irrotational, incompressible fluids. The lower (respectively, upper) fluid is denoted by a subscript 1 (respectively, 2 ). The one-dimensional free surface is $(x(\alpha, t), y(\alpha, t))$, where $\alpha$ is the parameter along the curve, and $t$ is time. We take the curve to be $2 \pi$-periodic, so that

$$
x(\alpha+2 \pi, t)=x(\alpha, t)+2 \pi, \quad y(\alpha+2 \pi, t)=y(\alpha, t),
$$


for all $\alpha$ and $t$. We let $\hat{\mathbf{t}}$ and $\hat{\mathbf{n}}$ be the unit tangent and normal vectors along the curve, defined as

$$
\hat{\mathbf{t}}=\frac{\left(x_{\alpha}, y_{\alpha}\right)}{s_{\alpha}}, \quad \hat{\mathbf{n}}=\frac{\left(-y_{\alpha}, x_{\alpha}\right)}{s_{\alpha}},
$$

with the arclength element, $s_{\alpha}$ defined by

$$
s_{\alpha}^{2}=x_{\alpha}^{2}+y_{\alpha}^{2} .
$$

We let $U$ and $V$ denote the normal and tangential velocities of the free surface, so that

$$
(x, y)_{t}=U \hat{\mathbf{n}}+V \hat{\mathbf{t}} .
$$

We introduce $\theta$, the tangent angle that the curve forms with the horizontal, defined as $\theta=$ $\tan ^{-1}\left(y_{\alpha} / x_{\alpha}\right)$. We can infer evolution equations for $s_{\alpha}$ and $\theta$ from (10) [25]; we find

$$
\begin{aligned}
& s_{\alpha, t}=V_{\alpha}-\theta_{\alpha} U, \\
& \theta_{t}=\frac{U_{\alpha}+V \theta_{\alpha}}{s_{\alpha}} .
\end{aligned}
$$

We make note of the following geometric identities:

$$
\hat{\mathbf{t}}_{\alpha}=\theta_{\alpha} \cdot \hat{\mathbf{n}}, \quad \hat{\mathbf{n}}_{\alpha}=-\theta_{\alpha} \cdot \hat{\mathbf{t}} .
$$

Of course, we also have the relationship between curvature and tangent angle:

$$
\kappa=\frac{\theta_{\alpha}}{s_{\alpha}} .
$$

While the normal velocity is dictated by the physics of the problem, the tangential velocity is not. That is to say, changing the tangential velocity only changes the parameterization of the interface, and so we may use the tangential velocity to enforce our preferred parameterization. Our preferred parameterization is a normalized arclength parameterization: we would like $s_{\alpha}$ to be independent of $\alpha$. If we let $L(t)$ denote the length of one period of the curve, then we would like $s_{\alpha, t}(\alpha, t)=L(t) / 2 \pi$ for all $t$. If this equation holds at the initial time, then it will hold at later times as long as

$$
s_{\alpha, t}=\frac{L_{t}}{2 \pi} .
$$

Since $L(t)=\int_{0}^{2 \pi} s_{\alpha}(\alpha, t) d \alpha$, we see from (11) using the periodicity of $V(\alpha, t)$ that

$$
L_{t}=-\int_{0}^{2 \pi} \theta_{\alpha} U d \alpha .
$$

Considering again (11), this implies

$$
V_{\alpha}=\frac{L_{t}}{2 \pi}+\theta_{\alpha} U=\mathbb{P}\left(\theta_{\alpha} U\right) .
$$

Integrating, we thus have

$$
V=\partial_{\alpha}^{-1} \mathbb{P}\left(\theta_{\alpha} U\right)+V(0, t) .
$$


The integration constant $V(0, t)$ is later chosen so that the mean of $V$ is the same as the mean of $\mathbf{W} \cdot \hat{\mathbf{t}}$.

Since there is no vorticity in the bulk of the fluid, we are able to use a vortex sheet formulation. The average of the upper and lower fluid velocities evlauated at the interface $S$ is denoted by $\mathbf{W}=\left(W_{1}, W_{2}\right)$ and is specified by the Birkhoff-Rott integral. In terms of the complex notation $(x, y) \rightarrow x+i y$, the Birkhoff-Rott integral is given by

$$
W_{1}-i W_{2}=\frac{1}{4 \pi i} \mathrm{PV} \int_{0}^{2 \pi} \gamma\left(\alpha^{\prime}\right) \cot \left(\frac{1}{2}\left(z(\alpha)-z\left(\alpha^{\prime}\right)\right)\right) d \alpha^{\prime} .
$$

The curve $z$ is the complex form of the interface $(x, y)$ :

$$
z(\alpha, t)=x(\alpha, t)+i y(\alpha, t) .
$$

The normal velocity is the normal component of the Birkhoff-Rott integral:

$$
U=\mathbf{W} \cdot \hat{\mathbf{n}} .
$$

The function $\gamma$ is the vortex sheet strength; it is the jump in velocity (lower minus upper fluid) across the interface. Since the velocity potential on each side of the interface satisfies a Bernoulli equation, upon taking the limit at the interface, an evolution equation for the jump in potential across the interface can be found. Differentiating this leads to the following evolution equation for $\gamma:$

$$
\gamma_{t}=-\frac{2}{\rho_{1}+\rho_{2}}[p]_{\alpha}+\frac{2 \pi}{L}((V-\mathbf{W} \cdot \hat{\mathbf{t}}) \gamma)_{\alpha}-2 A\left(\frac{L}{2 \pi} \mathbf{W}_{t} \cdot \hat{\mathbf{t}}+\frac{\pi^{2}}{L^{2}} \gamma \gamma_{\alpha}-(V-\mathbf{W} \cdot \hat{\mathbf{t}}) \mathbf{W}_{\alpha} \cdot \hat{\mathbf{t}}+g y_{\alpha}\right) .
$$

where $[p]=\left(p_{1}-p_{2}\right) \|_{S}$ is the jump in pressure at the interface and $g$ is the acceleration due to gravity. For details of the derivation of (18), the reader could consult [7] or [10] . The jump in pressure across the interface is given by (see $\S 8$ )

$$
[p]=E_{b}\left(\kappa_{s s}+\frac{\kappa^{3}}{2}-c(t) \kappa\right)
$$

In the above, $\rho_{1}$ and $\rho_{2}$ are the densities of fluid 1 and fluid 2, respectively. The Atwood number, $A$, is $A=\frac{\rho_{1}-\rho_{2}}{\rho_{1}+\rho_{2}}$, and $E_{b}$ is the bending modulus.

We present a nondimensionalized version of the equation for $\gamma$. Lengths are nondimensionalized by a representative length of the periodic domain $l$, pressure is made dimensionless by $E_{B} / l^{3}$, the surface tension parameter $c(t)$ by $E_{B} / l^{2}$, velocity by $\sqrt{l g}, \gamma$ by $l \sqrt{g}$, and time by $\sqrt{l / g}$. Introduce the dimensionless parameter

$$
S=\frac{E_{B}}{\left(\rho_{1}+\rho_{2}\right) l g} .
$$

The nondimensional equation for $\gamma$ is then

$$
\begin{aligned}
& \gamma_{t}=S\left(-\frac{\kappa_{\alpha \alpha}}{s_{\alpha}^{2}}-\frac{\kappa^{3}}{2}+\bar{c}_{1} \kappa\right)_{\alpha}+\frac{((V-\mathbf{W} \cdot \hat{\mathbf{t}}) \gamma)_{\alpha}}{s_{\alpha}} \\
&-2 A\left[\left(\mathbf{W}_{t} \cdot \hat{\mathbf{t}}\right) s_{\alpha}-(V-\mathbf{W} \cdot \hat{\mathbf{t}}) \mathbf{W}_{\alpha} \cdot \hat{\mathbf{t}}+\frac{1}{8} \partial_{\alpha}\left(\frac{\gamma^{2}}{s_{\alpha}^{2}}\right)+y_{\alpha}\right] .
\end{aligned}
$$


If we use the relationship $\kappa=\theta_{\alpha} / s_{\alpha}$, and distribute the derivative on the first term on the right-hand side, this is

$$
\begin{aligned}
\gamma_{t}=S\left(-\frac{\partial_{\alpha}^{4} \theta}{s_{\alpha}^{3}}-\frac{3 \theta_{\alpha}^{2} \theta_{\alpha \alpha}}{2 s_{\alpha}^{3}}+\bar{c}_{1} \frac{\theta_{\alpha \alpha}}{s_{\alpha}}\right)+ & \frac{((V-\mathbf{W} \cdot \hat{\mathbf{t}}) \gamma)_{\alpha}}{s_{\alpha}} \\
& -2 A\left[\left(\mathbf{W}_{t} \cdot \hat{\mathbf{t}}\right) s_{\alpha}-(V-\mathbf{W} \cdot \hat{\mathbf{t}}) \mathbf{W}_{\alpha} \cdot \hat{\mathbf{t}}+\frac{1}{8} \partial_{\alpha}\left(\frac{\gamma^{2}}{s_{\alpha}^{2}}\right)+y_{\alpha}\right] .
\end{aligned}
$$

\subsection{Approximating the Birkhoff-Rott integral}

We introduce a bit of notation which is helpful to us as we switch back forth between real and complex notation. We let $\Phi: \mathbb{R}^{2} \rightarrow \mathbb{C}$ be the complexification map,

$$
\Phi(a, b)=a+i b .
$$

Thus, for instance, $z=\Phi(x, y)$. We will denote the complex conjugate with ${ }^{*}$, as in $z^{*}=x-i y$.

We introduced previously the periodic Hilbert transform, $H$, but we only discussed it in terms of its symbol. There is an integral form of the Hilbert transform; if $f \in L^{2}$, say, then

$$
H f(\alpha)=\frac{1}{2 \pi} \mathrm{PV} \int_{0}^{2 \pi} f\left(\alpha^{\prime}\right) \cot \left(\frac{1}{2}\left(\alpha-\alpha^{\prime}\right)\right) d \alpha^{\prime} .
$$

We notice that (16), the formula for the Birkhoff-Rott integral, looks something like the Hilbert transform. We thus introduce the following operator, which is the error in approximating an integral like (16) with a Hilbert transform:

$$
\mathcal{K}\left[z_{d}\right] f(\alpha)=\frac{1}{4 \pi i} \int_{0}^{2 \pi} f\left(\alpha^{\prime}\right)\left[\cot \left(\frac{1}{2}\left(z_{d}(\alpha)-z_{d}\left(\alpha^{\prime}\right)\right)\right)-\frac{1}{z_{\alpha}\left(\alpha^{\prime}\right)} \cot \left(\frac{1}{2}\left(\alpha-\alpha^{\prime}\right)\right)\right] d \alpha^{\prime} .
$$

Here, we have introduced the quantity $z_{d}$, which is defined as

$$
z_{d}(\alpha, t)=z(\alpha, t)-z(0, t)
$$

we have already used in (21) the fact that $z(\alpha, t)-z\left(\alpha^{\prime}, t\right)=z_{d}(\alpha, t)-z_{d}\left(\alpha^{\prime}, t\right)$. It is convenient to use $z_{d}$ instead of $z$ because $z_{d}$ is determined uniquely from $\theta$, while $z$ is not. Notice furthermore that $\partial_{\alpha} z=\partial_{\alpha} z_{d}$. We will also need the commutator of the Hilbert transform with multiplication by a smooth function:

$$
[H, \phi] f(\alpha)=H(\phi f)(\alpha)-\phi(\alpha) H(f)(\alpha) .
$$

The operators $\mathcal{K}\left[z_{d}\right]$ and $[H, \phi]$ are both smoothing operators; estimates demonstrating this smoothing will be given in Section 4 below.

We will not provide the full details here, but having introduced these operators, we are able to write $\mathbf{W}_{\alpha}$ as follows:

$$
\mathbf{W}_{\alpha}=\frac{\pi}{L} H\left(\gamma_{\alpha}\right) \hat{\mathbf{n}}-\frac{\pi}{L} H\left(\gamma \theta_{\alpha}\right) \hat{\mathbf{t}}+\mathbf{m},
$$

where $\mathbf{m}$ is a collection of smoother terms given by

$$
\Phi(\mathbf{m})^{*}=z_{\alpha} \mathcal{K}\left[z_{d}\right]\left(\left(\frac{\gamma}{z_{\alpha}}\right)_{\alpha}\right)+\frac{z_{\alpha}}{2 i}\left[H, \frac{1}{z_{\alpha}^{2}}\right]\left(z_{\alpha}\left(\frac{\gamma}{z_{\alpha}}\right)_{\alpha}\right) .
$$


Formulas (22) and (23) were initially developed by the first author in [3], and used subsequently in several papers, including most recently [5].

We now give a useful formula for $V-\mathbf{W} \cdot \hat{\mathbf{t}}$. Notice that we can use $(13)$ to find that $(\mathbf{W} \cdot \hat{\mathbf{t}})_{\alpha}=$ $\mathbf{W}_{\alpha} \cdot \hat{\mathbf{t}}+(\mathbf{W} \cdot \hat{\mathbf{n}}) \theta_{\alpha}$. Since $V_{\alpha}=\frac{L_{t}}{2 \pi}+\theta_{\alpha} U$, and since $U=\mathbf{W} \cdot \hat{\mathbf{n}}$, we see that

$$
(V-\mathbf{W} \cdot \hat{\mathbf{t}})_{\alpha}=-\mathbf{W}_{\alpha} \cdot \hat{\mathbf{t}}+\frac{L_{t}}{2 \pi} .
$$

We substitute from $(22)$ to find

$$
(V-\mathbf{W} \cdot \hat{\mathbf{t}})_{\alpha}=\frac{\pi}{L} H\left(\gamma \theta_{\alpha}\right)-\mathbf{m} \cdot \hat{\mathbf{t}}+\frac{L_{t}}{2 \pi} .
$$

This can be rewritten by using the operator $\mathbb{P}$; note that the left-hand side has no mean, and that a Hilbert transform has no mean. Thus, we have the following:

$$
(V-\mathbf{W} \cdot \hat{\mathbf{t}})_{\alpha}=\frac{\pi}{L} H\left(\gamma \theta_{\alpha}\right)-\mathbb{P}(\mathbf{m} \cdot \hat{\mathbf{t}})
$$

We apply the operator $\partial_{\alpha}^{-1}$, and we also introduce the notation $V_{W}=V-\mathbf{W} \cdot \hat{\mathbf{t}}:$ get

$$
V_{W}=\partial_{\alpha}^{-1}\left(\frac{\pi}{L} H\left(\gamma \theta_{\alpha}\right)-\mathbb{P}(\mathbf{m} \cdot \hat{\mathbf{t}})\right)
$$

We note that it is implicit in this that the mean of $V$ is chosen to be the same as the mean of $\mathbf{W} \cdot \hat{\mathbf{t}}$; this is possible since the equation defining $V$ is an equation for $V_{\alpha}$, and the mean of $V$ is then free to be chosen.

We close this section with an expression for $\theta_{t}$. We start from (12), and we use the equation $U=\mathbf{W} \cdot \hat{\mathbf{n}}$ to find

$$
U_{\alpha}=\mathbf{W}_{\alpha} \cdot \hat{\mathbf{n}}-(\mathbf{W} \cdot \hat{\mathbf{t}}) \theta_{\alpha}
$$

Together with (22), this implies

$$
\theta_{t}=\frac{2 \pi^{2}}{L^{2}} H\left(\gamma_{\alpha}\right)+\frac{2 \pi}{L} V_{W} \theta_{\alpha}+\frac{2 \pi}{L} \mathbf{m} \cdot \hat{\mathbf{n}} .
$$

\subsection{Calculation of $\left(\mathbf{W}_{t} \cdot \hat{\mathbf{t}}\right) s_{\alpha}$}

In this section, we will rewrite $\left(\mathbf{W}_{t} \cdot \hat{\mathbf{t}}\right) s_{\alpha}$, which appears on the right-hand side of (20). To begin, we can write $\mathbf{W}_{t}$ as

$$
\begin{aligned}
\Phi\left(\mathbf{W}_{t}^{*}\right)=\frac{1}{4 \pi i} \mathrm{PV} \int \gamma_{t}\left(\alpha^{\prime}\right) \cot ( & \left.\frac{1}{2}\left(z(\alpha)-z\left(\alpha^{\prime}\right)\right)\right) d \alpha^{\prime} \\
& -\frac{1}{8 \pi i} \mathrm{PV} \int \gamma\left(\alpha^{\prime}\right)\left(z_{t}(\alpha)-z_{t}\left(\alpha^{\prime}\right)\right) \csc ^{2}\left(\frac{1}{2}\left(z(\alpha)-z\left(\alpha^{\prime}\right)\right)\right) d \alpha^{\prime} .
\end{aligned}
$$

We then write $\left(\mathbf{W}_{t} \cdot \hat{\mathbf{t}}\right) s_{\alpha}$ as

$$
\left(\mathbf{W}_{t} \cdot \hat{\mathbf{t}}\right) s_{\alpha}=\mathcal{J}\left[z_{d}\right]\left(\gamma_{t}\right)+R
$$


with the operator $\mathcal{J}\left[z_{d}\right]$ defined by

$$
\mathcal{J}\left[z_{d}\right](f)(\alpha)=\operatorname{Re}\left\{\frac{z_{\alpha}}{4 \pi i} \mathrm{PV} \int f\left(\alpha^{\prime}\right) \cot \left(\frac{1}{2}\left(z(\alpha)-z\left(\alpha^{\prime}\right)\right)\right) d \alpha^{\prime}\right\},
$$

and the term $R$ is given by

$$
R=\operatorname{Re}\left\{-\frac{z_{\alpha}}{8 \pi i} \operatorname{PV} \int \gamma\left(\alpha^{\prime}\right)\left(z_{t}(\alpha)-z_{t}\left(\alpha^{\prime}\right)\right) \csc ^{2}\left(\frac{1}{2}\left(z(\alpha)-z\left(\alpha^{\prime}\right)\right)\right) d \alpha^{\prime}\right\} .
$$

We will continue to rewrite $R$. To begin, we put in some factors of $z_{\alpha}\left(\alpha^{\prime}\right)$ and recognize a perfect derivative:

$$
\begin{aligned}
R=\operatorname{Re}\left\{-\frac{z_{\alpha}}{8 \pi i} \mathrm{PV}\right. & \left.\int \frac{\gamma\left(\alpha^{\prime}\right)}{z_{\alpha}\left(\alpha^{\prime}\right)}\left(z_{t}(\alpha)-z_{t}\left(\alpha^{\prime}\right)\right) z_{\alpha}\left(\alpha^{\prime}\right) \csc ^{2}\left(\frac{1}{2}\left(z(\alpha)-z\left(\alpha^{\prime}\right)\right)\right) d \alpha^{\prime}\right\} \\
& =\operatorname{Re}\left\{-\frac{z_{\alpha}}{4 \pi i} \mathrm{PV} \int \frac{\gamma\left(\alpha^{\prime}\right)}{z_{\alpha}\left(\alpha^{\prime}\right)}\left(z_{t}(\alpha)-z_{t}\left(\alpha^{\prime}\right)\right) \partial_{\alpha^{\prime}}\left(\cot \left(\frac{1}{2}\left(z(\alpha)-z\left(\alpha^{\prime}\right)\right)\right)\right) d \alpha^{\prime}\right\} .
\end{aligned}
$$

We then integrate by parts:

$$
R=\operatorname{Re}\left\{\frac{z_{\alpha}}{4 \pi i} \mathrm{PV} \int \partial_{\alpha^{\prime}}\left(\frac{\gamma\left(\alpha^{\prime}\right)\left(z_{t}(\alpha)-z_{t}\left(\alpha^{\prime}\right)\right)}{z_{\alpha}\left(\alpha^{\prime}\right)}\right) \cot \left(\frac{1}{2}\left(z(\alpha)-z\left(\alpha^{\prime}\right)\right)\right) d \alpha^{\prime}\right\}
$$

We apply the derivative, and write $R=R_{1}+R_{2}$, where $R_{1}$ and $R_{2}$ are given by the following formulas:

$$
\begin{gathered}
R_{1}=\operatorname{Re}\left\{-\frac{z_{\alpha}}{4 \pi i} \mathrm{PV} \int \frac{\gamma\left(\alpha^{\prime}\right) z_{t \alpha}\left(\alpha^{\prime}\right)}{z_{\alpha}\left(\alpha^{\prime}\right)} \cot \left(\frac{1}{2}\left(z(\alpha)-z\left(\alpha^{\prime}\right)\right)\right) d \alpha^{\prime}\right\}, \\
R_{2}=\operatorname{Re}\left\{\frac{z_{\alpha}}{4 \pi i} \operatorname{PV} \int \partial_{\alpha^{\prime}}\left(\frac{\gamma\left(\alpha^{\prime}\right)}{z_{\alpha}\left(\alpha^{\prime}\right)}\right)\left(z_{t}(\alpha)-z_{t}\left(\alpha^{\prime}\right)\right) \cot \left(\frac{1}{2}\left(z(\alpha)-z\left(\alpha^{\prime}\right)\right)\right) d \alpha^{\prime}\right\} .
\end{gathered}
$$

Of these, we need to continue to rewrite $R_{1}$, since it has significant terms we need to treat carefully. For $R_{2}$, we need to rewrite it in order to see that it contains no such significant terms. We treat $R_{2}$ first, by using $\mathcal{K}$ :

$$
R_{2}=-\operatorname{Re}\left\{\frac{z_{\alpha}}{2 i}\left[H, z_{t}\right]\left(\frac{1}{z_{\alpha}}\left(\frac{\gamma}{z_{\alpha}}\right)_{\alpha}\right)\right\}+\operatorname{Re}\left\{z_{\alpha} z_{t} \mathcal{K}\left[z_{d}\right]\left(\left(\frac{\gamma}{z_{\alpha}}\right)_{\alpha}\right)\right\}-\operatorname{Re}\left\{z_{\alpha} \mathcal{K}\left[z_{d}\right]\left(z_{t}\left(\frac{\gamma}{z_{\alpha}}\right)_{\alpha}\right)\right\} .
$$

We add and subtract in $R_{1}$ :

$$
R_{1}=\operatorname{Re}\left\{-\frac{z_{\alpha}}{2 i} H\left(\frac{\gamma z_{t \alpha}}{z_{\alpha}^{2}}\right)\right\}-\operatorname{Re}\left\{z_{\alpha} \mathcal{K}\left[z_{d}\right]\left(\frac{\gamma z_{t \alpha}}{z_{\alpha}}\right)\right\}
$$

To extract the important terms from this, we need a useful expression for $z_{t \alpha}$. We know that $z_{\alpha}=s_{\alpha} e^{i \theta}$ and $s_{\alpha}=L / 2 \pi$, so we have

$$
z_{t \alpha}=\frac{L_{t}}{2 \pi} e^{i \theta}+i \theta_{t} z_{\alpha}=\frac{L_{t}}{L} z_{\alpha}+i \theta_{t} z_{\alpha} .
$$


Using this, we have the following for $R_{1}$ :

$$
R_{1}=\operatorname{Re}\left\{-\frac{z_{\alpha}}{2} H\left(\frac{\gamma \theta_{t}}{z_{\alpha}}\right)\right\}+\operatorname{Re}\left\{-\frac{z_{\alpha} L_{t}}{2 L i} H\left(\frac{\gamma}{z_{\alpha}}\right)\right\}-\operatorname{Re}\left\{z_{\alpha} \mathcal{K}\left[z_{d}\right]\left(\frac{\gamma z_{t \alpha}}{z_{\alpha}}\right)\right\} .
$$

To continue, we substitute for $\theta_{t}$ from (25):

$$
\begin{aligned}
R_{1}=\operatorname{Re}\left\{-\frac{\pi^{2} z_{\alpha}}{L^{2}} H\left(\frac{\gamma H\left(\gamma_{\alpha}\right)}{z_{\alpha}}\right)\right\}+\operatorname{Re}\left\{-\frac{\pi z_{\alpha}}{L} H\left(\frac{\gamma V_{W} \theta_{\alpha}}{z_{\alpha}}\right)\right\}+\operatorname{Re}\left\{-\frac{\pi z_{\alpha}}{L} H\left(\frac{\gamma \mathbf{m} \cdot \hat{\mathbf{n}}}{z_{\alpha}}\right)\right\} \\
+\operatorname{Re}\left\{-\frac{z_{\alpha} L_{t}}{2 L i} H\left(\frac{\gamma}{z_{\alpha}}\right)\right\}-\operatorname{Re}\left\{z_{\alpha} \mathcal{K}\left[z_{d}\right]\left(\frac{\gamma z_{t \alpha}}{z_{\alpha}}\right)\right\} .
\end{aligned}
$$

Next, for the first two terms on the right-hand side of (27), we pull some things through the Hilbert transform, incurring commutators in the process:

$$
\begin{array}{r}
R_{1}=\frac{\pi^{2}}{L^{2}} \gamma \gamma_{\alpha}-\frac{\pi}{L} V_{W} H\left(\gamma \theta_{\alpha}\right)+\operatorname{Re}\left\{-\frac{\pi^{2} z_{\alpha}}{L^{2}}\left[H, \frac{\gamma}{z_{\alpha}}\right]\left(H\left(\gamma_{\alpha}\right)\right)\right\}+\operatorname{Re}\left\{-\frac{\pi z_{\alpha}}{L}\left[H, \frac{V_{W}}{z_{\alpha}}\right]\left(\gamma \theta_{\alpha}\right)\right\} \\
+\operatorname{Re}\left\{-\frac{\pi z_{\alpha}}{L} H\left(\frac{\gamma \mathbf{m} \cdot \hat{\mathbf{n}}}{z_{\alpha}}\right)\right\}+\operatorname{Re}\left\{-\frac{z_{\alpha} L_{t}}{2 L i} H\left(\frac{\gamma}{z_{\alpha}}\right)\right\}-\operatorname{Re}\left\{z_{\alpha} \mathcal{K}\left[z_{d}\right]\left(\frac{\gamma z_{t \alpha}}{z_{\alpha}}\right)\right\} .
\end{array}
$$

We give the name $R_{3}$ to the sum of the last four terms on the right-hand side of (28), so that we have

$$
R_{1}=\frac{\pi^{2}}{L^{2}} \gamma \gamma_{\alpha}-\frac{\pi}{L} V_{W} H\left(\gamma \theta_{\alpha}\right)+R_{3}
$$

The conclusion of this subsection is the following formula:

$$
\left(\mathbf{W}_{t} \cdot \hat{\mathbf{t}}\right) s_{\alpha}=-\frac{\pi}{L} V_{W} H\left(\gamma \theta_{\alpha}\right)+\frac{\pi^{2}}{L^{2}} \gamma \gamma_{\alpha}+\mathcal{J}\left[z_{d}\right] \gamma_{t}+R_{2}+R_{3} .
$$

We will estimate $\mathcal{J}\left[z_{d}\right]\left(\gamma_{t}\right), R_{2}$, and $R_{3}$ in Section 4 below.

\subsection{Our small-scale decomposition}

We are now going to rewrite the above evolution equations in an important ways: we will emphasize the terms in the $\theta_{t}$ and $\gamma_{t}$ evolution equations which must be treated carefully in the energy estimates.

We pick up from (20), and we replace $s_{\alpha}$ with $L / 2 \pi$. We also introduce the notation

$$
\widetilde{S}(t)=\frac{S}{s_{\alpha}^{3}}=\frac{8 \pi^{3} S}{L^{3}}
$$

We also apply the $\alpha$-derivative in the second term on the right hand side in (20). These considerations yield the following:

$$
\begin{aligned}
\gamma_{t}=\widetilde{S}\left(-\partial_{\alpha}^{4} \theta-\theta_{\alpha \alpha}\left(\frac{3}{2} \theta_{\alpha}^{2}-\frac{L^{2} \bar{c}_{1}}{4 \pi^{2}}\right)\right)+\frac{2 \pi \gamma \partial_{\alpha} V_{W}}{L} & +\frac{2 \pi V_{W} \gamma_{\alpha}}{L} \\
-2 A & {\left[\left(\mathbf{W}_{t} \cdot \hat{\mathbf{t}}\right) s_{\alpha}-V_{W} \mathbf{W}_{\alpha} \cdot \hat{\mathbf{t}}+\frac{1}{8} \partial_{\alpha}\left(\frac{4 \pi^{2} \gamma^{2}}{L^{2}}\right)-y_{\alpha}\right] . }
\end{aligned}
$$


Next, we substitute for both $\left(\mathbf{W}_{t} \cdot \hat{\mathbf{t}}\right) s_{\alpha}$ from (30) and for $\mathbf{W}_{\alpha} \cdot \hat{\mathbf{t}}$, using (22). In doing so, there is an important cancellation, since both of these terms contribute a term $\frac{\pi}{L} V_{W} H\left(\gamma \theta_{\alpha}\right)$, with opposite signs. After making this cancellation, we are left with

$$
\begin{array}{r}
\gamma_{t}=\widetilde{S}\left(-\partial_{\alpha}^{4} \theta-\theta_{\alpha \alpha}\left(\frac{3}{2} \theta_{\alpha}^{2}-\frac{L^{2} \bar{c}_{1}}{4 \pi^{2}}\right)\right)+\frac{2 \pi \gamma \partial_{\alpha} V_{W}}{L}+\frac{2 \pi V_{W} \gamma_{\alpha}}{L} \\
-2 A\left[\mathcal{J}\left[z_{d}\right] \gamma_{t}+R_{2}+R_{3}-V_{W} \mathbf{m} \cdot \hat{\mathbf{t}}+\frac{\pi^{2}}{L^{2}} \gamma \gamma_{\alpha}-y_{\alpha}\right]
\end{array}
$$

which is an integral equation for $\gamma_{t}$. Finally, we group together some like terms, writing the integral equation above as

$$
\gamma_{t}=-\widetilde{S} \partial_{\alpha}^{4} \theta-\widetilde{S} \theta_{\alpha \alpha} Q_{1}+\gamma_{\alpha} Q_{2}+Q_{3},
$$

where $Q_{1}, Q_{2}$, and $Q_{3}$ are the following collections of smoother terms:

$$
\begin{gathered}
Q_{1}:=Q_{1}(\alpha, t)=\frac{3}{2} \theta_{\alpha}^{2}-\frac{L^{2} \bar{c}_{1}}{4 \pi^{2}} \\
Q_{2}:=Q_{2}(\alpha, t)=\frac{2 \pi V_{W}}{L}-\frac{2 A \pi^{2}}{L^{2}} \gamma, \\
Q_{3}:=Q_{3}(\alpha, t)=\frac{2 \pi \gamma \partial_{\alpha} V_{W}}{L}-2 A \mathcal{J}\left[z_{d}\right] \gamma_{t}-2 A R_{2}-2 A R_{3}+2 A V_{W} \mathbf{m} \cdot \hat{\mathbf{t}}-2 A y_{\alpha} .
\end{gathered}
$$

It will be helpful to have a brief notation for the evolution equations, so we introduce the following:

$$
(\theta, \gamma)_{t}=\mathcal{B}=\left(\mathcal{B}_{1}, \mathcal{B}_{2}\right)
$$

The definitions of $\mathcal{B}_{1}$ and $\mathcal{B}_{2}$ are clearly just given by the right-hand sides of the the equations (25) and (31).

\section{Preliminary estimates}

We present without proof several important lemmas that are used in the subsequent estimates. The proofs of Lemmas 1 through 5 can be found in [3]. A version of Lemma 6 also appears in [3], but the particular form of the statement and proof of Lemma 6 is from [18] (see also [10] and [41]). Versions of many of these lemmas may also be found in [6] or [11].

The first lemma is a standard interpolation lemma for Sobolev spaces:

Lemma 1 Let $m \geq 0$ and $\ell \geq m$ be given. Let $f \in H^{\ell}$ be given. Then, the following inequality holds:

$$
\|f\|_{m} \leq c\|f\|_{\ell}^{m / \ell}\|f\|_{0}^{1-m / \ell}
$$

The remainder operator $\mathcal{K}$ is a smoothing operator; the following lemma makes this precise. Establishing this requires an assumption on the chord arc quantity

$$
q_{1}\left(\alpha, \alpha^{\prime}\right)=\frac{z_{d}(\alpha)-z_{d}\left(\alpha^{\prime}\right)}{\alpha-\alpha^{\prime}}
$$

which states that the interfacial curve $z_{d}$ is not close to self-intersecting. 
Lemma 2 Let $n \geq 2$ be an integer. Assume $z_{d} \in H^{n}$. Assume there exists $\beta_{1}>0$ such that for all $\alpha$ and $\alpha^{\prime}$,

$$
\left|q_{1}\left(\alpha, \alpha^{\prime}\right)\right|>\beta_{1} .
$$

Then $\mathcal{K}\left[z_{d}\right]: H^{1} \rightarrow H^{n-1}$ and $\mathcal{K}\left[z_{d}\right]: H^{0} \rightarrow H^{n-2}$, with the estimates

$$
\begin{gathered}
\left\|\mathcal{K}\left[z_{d}\right] f\right\|_{n-1} \leq C_{1}\|f\|_{1} \exp \left\{C_{2}\left\|z_{d}\right\|_{n}\right\}, \\
\left\|\mathcal{K}\left[z_{d}\right] f\right\|_{n-2} \leq C_{1}\|f\|_{0} \exp \left\{C_{2}\left\|z_{d}\right\|_{n}\right\} .
\end{gathered}
$$

We also will need a Lipschitz estimate for $\mathcal{K}$, when we establish uniqueness and continuous dependence of solutions.

Lemma 3 Let $\theta$ and $\theta^{\prime}$ be in $H^{3}$. Let $L$ and $L^{\prime}$ be the corresponding lengths of the associated curves $z_{d}$ and $z_{d}^{\prime}$, and let $q_{1}$ and $q_{1}^{\prime}$ be the associated chord-arc quantities. Assume there exists positive constants $\beta_{1}$ and $\beta_{2}$ such that $L<\beta_{2}$ and $L^{\prime}<\beta_{2}$, and for all $\alpha$ and $\alpha^{\prime}$,

$$
\left|q_{1}\left(\alpha, \alpha^{\prime}\right)\right|>\beta_{1}, \quad\left|q_{1}^{\prime}\left(\alpha, \alpha^{\prime}\right)\right|>\beta_{1} .
$$

Then the following Lipschitz estimate holds, for any $f \in H^{3}$ :

$$
\left\|\mathcal{K}\left[z_{d}\right] f-\mathcal{K}\left[z_{d}^{\prime}\right] f\right\|_{3} \leq c\left\|\theta-\theta^{\prime}\right\|_{3}\|f\|_{3} .
$$

A version of Lemma 3 was proved in [2] giving an estimate in $H^{1}$ rather than $H^{3}$. The same proof goes through, however, in $H^{3}$, so we omit it.

We have two different commutator estimates for the commutator of the Hilbert transform and multiplication by a smooth function. The first of these yields less regularity for the commutator, but requires less regularity on the functions.

Lemma 4 Let $n \geq 1$ be an integer. Let $\phi \in H^{n}$ be given. Then $[H, \phi]: H^{0} \rightarrow H^{n-1}$ and $[H, \phi]:$ $H^{-1} \rightarrow H^{n-2}$, with the estimates

$$
\begin{gathered}
\|[H, \phi] f\|_{n-1} \leq c\|\phi\|_{n}\|f\|_{0}, \\
\|[H, \phi] f\|_{n-2} \leq c\|\phi\|_{n}\|f\|_{-1} .
\end{gathered}
$$

Our second commutator lemma gives higher regularity of the commutator, by requiring more regularity on the functions.

Lemma 5 Let $j \geq 1$ be an integer. Let $n \geq 2 j$ be an integer. Let $\phi \in H^{n}$ be given. Then, $[H, \phi]$ : $H^{n-j} \rightarrow H^{n}$, with the estimate

$$
\|[H, \phi] f\|_{n} \leq c\|\phi\|_{n}\|f\|_{n-j} .
$$

We need a lemma giving solvability of our $\gamma_{t}$ integral equation (31).

Lemma 6 Assume $z_{d} \in H^{n}$ for $n \geq 3$. The operator $\left(I+2 A \mathcal{J}\left[z_{d}\right]\right)^{-1}$ is bounded from $H^{0}$ to $H^{0}$, with the estimate

$$
\left\|\left(I+2 A \mathcal{J}\left[z_{d}\right]\right)^{-1} F\right\|_{0} \leq c_{1} \exp \left\{c_{2}\left\|z_{d}\right\|_{3}\right\}\|F\|_{0} .
$$




\section{Existence}

Before proving existence of solutions, we first must introduce a regularized system of evolution equations. We will first prove existence of solutions for the regularized system, and then prove energy estimates for the regularized system. We will then be able to pass to the limit as the regularization parameter vanishes, finding that solutions of the non-regularized system of evolution equations exist.

\subsection{The mollified system}

We will need to be careful about reconstructing a curve from a tangent angle; this is because not every periodic tangent angle function will lead to a periodic curve. In particular, say $\eta$ is our tangent angle function, perhaps at a step of an iteration procedure (so that $\eta$ cannot be assumed to be a solution of our evolution equation). First we concern ourselves with defining the length of the curve; this comes from the horizontal periodicity.

The derivative of the horizontal component of the curve to be constructed from $\eta$ is

$$
x_{\alpha}[\eta, L]=\frac{L}{2 \pi} \cos (\eta) .
$$

The horizontal periodicity requires that $x[\eta, L](2 \pi)-x[\eta, L](0)=2 \pi$, so we have

$$
2 \pi=\int_{0}^{2 \pi} x_{\alpha}[\eta, L](\alpha) d \alpha=\frac{L}{2 \pi} \int_{0}^{2 \pi} \cos (\eta(\alpha)) d \alpha
$$

Solving for $L$, this is

$$
L[\eta]=\frac{4 \pi^{2}}{\int_{0}^{2 \pi} \cos (\eta(\alpha)) d \alpha} .
$$

For $y_{\alpha}[\eta, L]$, we want $\int_{0}^{2 \pi} y_{\alpha}[\eta, L](\alpha) d \alpha=0$. To enforce our periodicity condition on $x[\eta, L]$, we were able to choose $L[\eta]$ accordingly; there is no corresponding choice we can make in this case. Instead, we simply must project the mean away. We define $y_{\alpha}[\eta, L]$ to be

$$
y_{\alpha}[\eta]=\frac{L[\eta]}{2 \pi} \mathbb{P} \sin (\eta)
$$

where $\mathbb{P}$ is the projection which zeros out the mean.

We must define the mollified curve, and we use the above discussion as guidance. We let $\theta^{\varepsilon}$ be given. We let the length, $L^{\varepsilon}$, be defined as

$$
L^{\varepsilon}=L\left[\theta^{\varepsilon}\right] .
$$

Naturally, since $\widetilde{S}=\frac{8 \pi^{3} S}{L^{3}}$, we will use the notation $\widetilde{S}^{\varepsilon}$ :

$$
\widetilde{S}^{\varepsilon}=\frac{8 \pi^{3} S}{\left(L^{\varepsilon}\right)^{3}} .
$$

The derivative of the curve is given by

$$
x_{\alpha}^{\varepsilon}=x_{\alpha}\left[\theta^{\varepsilon}, L^{\varepsilon}\right]=\frac{L^{\varepsilon}}{2 \pi} \cos \left(\theta^{\varepsilon}\right),
$$




$$
y_{\alpha}^{\varepsilon}=y_{\alpha}\left[\theta^{\varepsilon}, L^{\varepsilon}\right]=\frac{L^{\varepsilon}}{2 \pi} \mathbb{P} \sin \left(\theta^{\varepsilon}\right) .
$$

The mollified curve is then defined by integrating:

$$
z_{d}^{\varepsilon}=\frac{L^{\varepsilon}}{2 \pi} \int_{0}^{\alpha} \cos \left(\theta^{\varepsilon}\right)+i \mathbb{P} \sin \left(\theta^{\varepsilon}\right) d \alpha
$$

and the unit normal and tangent curves are defined to be

$$
\hat{\mathbf{t}}^{\varepsilon}=\left(\cos \left(\theta^{\varepsilon}\right), \sin \left(\theta^{\varepsilon}\right)\right), \quad \hat{\mathbf{n}}^{\varepsilon}=\left(-\sin \left(\theta^{\varepsilon}\right), \cos \left(\theta^{\varepsilon}\right)\right) .
$$

(Note that if $\theta^{\varepsilon}$ does not satisfy $\mathbb{P} \sin \left(\theta^{\varepsilon}\right)=\sin \left(\theta^{\varepsilon}\right)$, then these vectors $\hat{\mathbf{t}}^{\varepsilon}$ and $\hat{\mathbf{n}}^{\varepsilon}$ are not actually the unit tangent and normal vectors to the curve $z_{d}^{\varepsilon}$. We will ensure that when $\theta^{\varepsilon}$ is a solution of of the evolution equation (to be defined), that the property $\mathbb{P} \sin \left(\theta^{\varepsilon}\right)=\sin \left(\theta^{\varepsilon}\right)$ holds.)

For the exact evolution equations, we have $\theta_{t}=\frac{U_{\alpha}+V \theta_{\alpha}}{s_{\alpha}}$. If we study $\int_{0}^{2 \pi} \sin (\theta(\alpha, t)) d \alpha$ in this case, we find

$$
\frac{d}{d t} \int_{0}^{2 \pi} \sin (\theta(\alpha, t)) d \alpha=\frac{1}{s_{\alpha}} \int_{0}^{2 \pi} \cos (\theta)\left(U_{\alpha}+V \theta_{\alpha}\right) d \alpha
$$

We notice that $\cos (\theta) \theta_{\alpha}=\partial_{\alpha} \sin (\theta)$, and we integrate by parts for this term. We also recall (14), which says that $V_{\alpha}=\frac{L_{t}}{2 \pi}+\theta_{\alpha} U$. These considerations yield the following:

$$
\frac{d}{d t} \int_{0}^{2 \pi} \sin (\theta(\alpha, t)) d \alpha=\frac{1}{s_{\alpha}} \int_{0}^{2 \pi}\left[\cos (\theta) U_{\alpha}-\sin (\theta) \theta_{\alpha} U\right] d \alpha-\frac{L_{t}}{L} \int_{0}^{2 \pi} \sin (\theta) d \alpha .
$$

Integrating by parts in the first term, we see that

$$
\frac{d}{d t} \int_{0}^{2 \pi} \sin (\theta(\alpha, t)) d \alpha=-\frac{L_{t}}{L} \int_{0}^{2 \pi} \sin (\theta) d \alpha .
$$

This implies that the mean of $\sin (\theta)$ grows or decays exponentially, with the exponential growth rate related to $L$ and $L_{t}$. Thus, for a solution, $\theta$, of the exact evolution equations, we see that if the mean of $\sin (\theta)$ is initially zero, then it will remain zero at positive times.

We introduce the following analogue of (32) for the mollified system:

$$
\left(\theta^{\varepsilon}, \gamma^{\varepsilon}\right)_{t}=\left(\mathcal{B}_{1}^{\varepsilon}+\mu^{\varepsilon}, \mathcal{B}_{2}^{\varepsilon}\right)
$$

where we must now define $\mathcal{B}_{1}^{\varepsilon}, \mathcal{B}_{2}^{\varepsilon}$, and $\mu^{\varepsilon}$. Of these, $\mathcal{B}_{1}^{\varepsilon}$ and $\mathcal{B}_{2}^{\varepsilon}$ will be rather clearly similar to $\mathcal{B}_{1}$ and $\mathcal{B}_{2}$, but with mollification operators applied in a variety of places. The other term, $\mu^{\varepsilon}$, will be used to enforce our periodicity requirement, that $\mathbb{P}\left(\sin \left(\theta^{\varepsilon}\right)\right)=\sin \left(\theta^{\varepsilon}\right)$. We remark that $\mu^{\varepsilon}$ is taken to be a function of $t$ only, and to not depend on $\alpha$; that is, $\mu^{\varepsilon}$ will be related to spatial averages of other quantities, and will thus be a constant function with respect to the spatial variable.

The previous argument, for the non-mollified system, showed that the structure of the nonmollified evolution equations implies $\frac{d}{d t} \int_{0}^{2 \pi} \sin (\theta(\alpha, t)) d \alpha=0$. For solutions of the mollified system, we no longer have the exact structure that we used previously, and we instead define $\mu^{\varepsilon}$ to achieve our desired goal. In particular, we have the following:

$$
\frac{d}{d t} \int_{0}^{2 \pi} \sin \left(\theta^{\varepsilon}\right) d \alpha=\int_{0}^{2 \pi} \theta_{t}^{\varepsilon} \cos \left(\theta^{\varepsilon}\right) d \alpha=\int_{0}^{2 \pi} \mathcal{B}_{1}^{\varepsilon} \cos \left(\theta^{\varepsilon}\right) d \alpha+\mu^{\varepsilon} \int_{0}^{2 \pi} \cos \left(\theta^{\varepsilon}\right) d \alpha .
$$


Since we want this to equal zero, we make the following definition of $\mu^{\varepsilon}$ :

$$
\mu^{\varepsilon}=-\frac{\int_{0}^{2 \pi} \mathcal{B}_{1}^{\varepsilon} \cos \left(\theta^{\varepsilon}\right) d \alpha}{\int_{0}^{2 \pi} \cos \left(\theta^{\varepsilon}\right) d \alpha}=-\frac{L^{\varepsilon}}{4 \pi^{2}} \int_{0}^{2 \pi} \mathcal{B}_{1}^{\varepsilon} \cos \left(\theta^{\varepsilon}\right) d \alpha .
$$

The point of this is that, if $\left(\theta^{\varepsilon}, \gamma^{\varepsilon}\right)$ solves (41), then $\mathbb{P}\left(\sin \left(\theta^{\varepsilon}\right)\right)=\sin \left(\theta^{\varepsilon}\right)$. In light of (35) and (36), this implies

$$
\left|z_{\alpha}^{\varepsilon}(\alpha, t)\right|=\frac{L^{\varepsilon}(t)}{2 \pi}, \quad \forall \alpha,
$$

as desired; this would not be the case if the mean of $\sin \left(\theta^{\varepsilon}\right)$ were nonzero.

We let the mollifier with parameter $\varepsilon$ be denoted $\chi_{\varepsilon}$; this operator acts through truncation of the Fourier series, zeroing out modes with wavenumber larger than $1 / \varepsilon$. As such, $\chi_{\varepsilon}$ is a projection, so that $\chi_{\varepsilon}^{2}=\chi_{\varepsilon}$.

We now define $\mathcal{B}_{1}^{\varepsilon}$ and $\mathcal{B}_{2}^{\varepsilon}$. To begin, we make the following definitions:

$$
\begin{aligned}
& \mathcal{B}_{1}^{\varepsilon}=\frac{2 \pi^{2}}{\left(L^{\varepsilon}\right)^{2}} \chi_{\varepsilon} H\left(\gamma_{\alpha}^{\varepsilon}\right)+\frac{2 \pi}{L^{\varepsilon}} \chi_{\varepsilon}\left(V_{W}^{\varepsilon}\left(\chi_{\varepsilon} \theta_{\alpha}^{\varepsilon}\right)\right)+\frac{2 \pi}{L^{\varepsilon}} \mathbf{m}^{\varepsilon} \cdot \hat{\mathbf{n}}^{\varepsilon}, \\
& \mathcal{B}_{2}^{\varepsilon}=-\widetilde{S}^{\varepsilon} \chi_{\varepsilon} \partial_{\alpha}^{4} \theta^{\varepsilon}-\widetilde{S}^{\varepsilon} \chi_{\varepsilon}\left(Q_{1}^{\varepsilon}\left(\chi_{\varepsilon} \theta_{\alpha \alpha}^{\varepsilon}\right)\right)+\chi_{\varepsilon}\left(Q_{2}^{\varepsilon}\left(\chi_{\varepsilon} \gamma_{\alpha}^{\varepsilon}\right)\right)+Q_{3}^{\varepsilon} .
\end{aligned}
$$

In some of the terms the mollification operator $\chi^{\varepsilon}$ appears twice; the reason for this is so that we can perform integration by parts in the energy estimate. The placement of mollifiers will become clear in the proof of Theorem 10. At this point, we have almost completely specified the mollified system. What remains now is to give the definition of some of the mollified versions of the auxiliary quantities, such as $V_{W}^{\varepsilon}$ and $Q_{1}^{\varepsilon}$, among others.

$$
\begin{gathered}
V_{W}^{\varepsilon}=\partial_{\alpha}^{-1}\left(\frac{\pi}{L^{\varepsilon}} H\left(\left(\chi_{\varepsilon} \gamma^{\varepsilon}\right)\left(\chi_{\varepsilon} \theta_{\alpha}^{\varepsilon}\right)\right)-\mathbb{P}\left(\mathbf{m}^{\varepsilon} \cdot \hat{\mathbf{t}}^{\varepsilon}\right)\right) . \\
Q_{1}^{\varepsilon}=\frac{3}{2}\left(\chi_{\varepsilon} \theta_{\alpha}^{\varepsilon}\right)^{2}-\frac{\left(L^{\varepsilon}\right)^{2} \bar{c}_{1}}{4 \pi^{2}}
\end{gathered}
$$

We define $\mathbf{m}^{\varepsilon}$ the same way that $\mathbf{m}$ is defined in (23), but we use the mollified quantities instead:

$$
\Phi\left(\mathbf{m}^{\varepsilon}\right)^{*}=z_{\alpha}^{\varepsilon} \mathcal{K}\left[z_{d}^{\varepsilon}\right]\left(\left(\frac{\gamma^{\varepsilon}}{z_{\alpha}^{\varepsilon}}\right)_{\alpha}\right)+\frac{z_{\alpha}^{\varepsilon}}{2 i}\left[H, \frac{1}{\left(z_{\alpha}^{\varepsilon}\right)^{2}}\right]\left(z_{\alpha}^{\varepsilon}\left(\frac{\gamma^{\varepsilon}}{z_{\alpha}^{\varepsilon}}\right)_{\alpha}\right) .
$$

The mollified Birkhoff-Rott integral, $\mathbf{W}^{\varepsilon}$, is similarly defined the same way as $\mathbf{W}$, but in terms of the new quantities. We have

$$
\Phi\left(\mathbf{W}^{\varepsilon}\right)^{*}=\frac{1}{4 \pi i} \mathrm{PV} \int_{0}^{2 \pi} \gamma^{\varepsilon}\left(\alpha^{\prime}\right) \cot \left(\frac{1}{2}\left(z_{d}^{\varepsilon}(\alpha)-z_{d}^{\varepsilon}\left(\alpha^{\prime}\right)\right)\right) d \alpha^{\prime} .
$$

Then, we define $U^{\varepsilon}$ to be

$$
U^{\varepsilon}=\mathbf{W}^{\varepsilon} \cdot \hat{\mathbf{n}}^{\varepsilon} .
$$

We also need the mollified version of (22)

$$
\mathbf{W}_{\alpha}^{\varepsilon}=\frac{\pi}{L^{\varepsilon}} H\left(\gamma_{\alpha}^{\varepsilon}\right) \hat{\mathbf{n}}^{\varepsilon}-\frac{\pi}{L^{\varepsilon}} H\left(\gamma^{\varepsilon} \theta_{\alpha}^{\varepsilon}\right) \hat{\mathbf{t}}^{\varepsilon}+\mathbf{m}^{\varepsilon},
$$


We let $V^{\varepsilon}$ be the mollified version of $V$, which was defined in (15):

$$
V^{\varepsilon}=\partial_{\alpha}^{-1} \mathbb{P}\left(\theta_{\alpha}^{\varepsilon} U^{\varepsilon}\right)+V^{\varepsilon}(0, t) .
$$

We next define $Q_{2}^{\varepsilon}$ and $Q_{3}^{\varepsilon}$. Of these, $Q_{2}^{\varepsilon}$ is straightforward and $Q_{3}^{\varepsilon}$ will take some effort. We let $Q_{2}^{\varepsilon}$ be given by

$$
Q_{2}^{\varepsilon}=\frac{2 \pi V_{W}^{\varepsilon}}{L^{\varepsilon}}-\frac{2 A \pi^{2}}{\left(L^{\varepsilon}\right)^{2}} \gamma^{\varepsilon} .
$$

To define $Q_{3}^{\varepsilon}$, we first need to rewrite, again, the $\gamma_{t}$ equation (to deal with the fact that it is actually an integral equation). We write it as

$$
\gamma_{t}=-2 A \mathcal{J}\left[z_{d}\right] \gamma_{t}+\Xi
$$

where

$$
\Xi=-\widetilde{S} \partial_{\alpha}^{4} \theta-\widetilde{S} Q_{1}+\gamma_{\alpha} Q_{2}+\widetilde{Q}_{3},
$$

with $\widetilde{Q}_{3}$ given by

$$
\widetilde{Q}_{3}=\frac{2 \pi \gamma \partial_{\alpha} V_{W}}{L}-2 A R_{2}-2 A R_{3}+2 A V_{W} \mathbf{m} \cdot \hat{\mathbf{t}}-2 A y_{\alpha} .
$$

Then, we can solve for $\gamma_{t}$ :

$$
\gamma_{t}=\left(I+2 A \mathcal{J}\left[z_{d}\right]\right)^{-1} \Xi
$$

We can then rewrite $Q_{3}$ as

$$
Q_{3}=\widetilde{Q}_{3}-2 A \mathcal{J}\left[z_{d}\right]\left(I+2 A \mathcal{J}\left[z_{d}\right]\right)^{-1} \Xi .
$$

We can then define $\widetilde{Q}_{3}^{\varepsilon}$ and $\Xi^{\varepsilon}$ :

$$
\begin{gathered}
\widetilde{Q}_{3}^{\varepsilon}=\frac{2 \pi \gamma^{\varepsilon} V_{W}^{\varepsilon}}{L^{\varepsilon}}-2 A R_{2}^{\varepsilon}-2 A R_{3}^{\varepsilon}+2 A V_{W}^{\varepsilon} \mathbf{m}^{\varepsilon} \hat{\mathbf{t}}^{\varepsilon}-2 A y_{\alpha}^{\varepsilon}, \\
\Xi^{\varepsilon}=-\widetilde{S}^{\varepsilon} \partial_{\alpha}^{4} \theta^{\varepsilon}-\widetilde{S}^{\varepsilon} Q_{1}^{\varepsilon}+\gamma_{\alpha}^{\varepsilon} Q_{2}^{\varepsilon}+\widetilde{Q}_{3}^{\varepsilon} .
\end{gathered}
$$

This, naturally, still leaves us needing to define $R_{2}^{\varepsilon}$ and $R_{3}^{\varepsilon}$; we will do this in a moment. First, however, we define $Q_{3}^{\varepsilon}$ as

$$
Q_{3}^{\varepsilon}=\widetilde{Q}_{3}^{\varepsilon}-2 A \mathcal{J}\left[z_{d}^{\varepsilon}\right]\left(I+2 A \mathcal{J}\left[z_{d}^{\varepsilon}\right]\right)^{-1} \Xi^{\varepsilon} .
$$

For $R_{3}$, the original statement of its definition involved $L_{t}$ and $z_{t \alpha}$; we now rewrite $R_{3}$ by substituting for these:

$$
\begin{aligned}
R_{3}=\operatorname{Re}\left\{-\frac{\pi z_{\alpha}}{L}\left[H, \frac{V_{W}}{z_{\alpha}}\right]\left(\gamma \theta_{\alpha}\right)\right\} & +\operatorname{Re}\left\{-\frac{\pi}{z_{\alpha}} H\left(\frac{\gamma \mathbf{m} \cdot \hat{\mathbf{n}}}{z_{\alpha}}\right)\right\} \\
+ & \operatorname{Re}\left\{\frac{z_{\alpha}\left(\int_{0}^{2 \pi} \theta_{\alpha} U d \alpha\right)}{2 L i} H\left(\frac{\gamma}{z_{\alpha}}\right)\right\}-\operatorname{Re}\left\{z_{\alpha} \mathcal{K}\left[z_{d}\right]\left(\frac{\gamma \partial_{\alpha}(U \hat{\mathbf{n}}+V \hat{\mathbf{t}}}{z_{\alpha}}\right)\right\} .
\end{aligned}
$$


Defining $R_{3}^{\varepsilon}$ is then straightforward:

$$
\begin{aligned}
R_{3}^{\varepsilon}=\operatorname{Re}\left\{-\frac{\pi z_{\alpha}^{\varepsilon}}{L^{\varepsilon}}\left[H, \frac{V_{W}^{\varepsilon}}{z_{\alpha}^{\varepsilon}}\right]\left(\gamma^{\varepsilon} \theta_{\alpha}^{\varepsilon}\right)\right\}+\operatorname{Re}\left\{-\frac{\pi}{z_{\alpha}^{\varepsilon}} H\left(\frac{\gamma^{\varepsilon} \mathbf{m}^{\varepsilon} \cdot \hat{\mathbf{n}}^{\varepsilon}}{z_{\alpha}^{\varepsilon}}\right)\right\} \\
+\operatorname{Re}\left\{\frac{z_{\alpha}^{\varepsilon}\left(\int_{0}^{2 \pi} \theta_{\alpha}^{\varepsilon} U^{\varepsilon} d \alpha\right)}{2 L^{\varepsilon} i} H\left(\frac{\gamma^{\varepsilon}}{z_{\alpha}^{\varepsilon}}\right)\right\}-\operatorname{Re}\left\{z_{\alpha}^{\varepsilon} \mathcal{K}\left[z_{d}^{\varepsilon}\right]\left(\frac{\gamma^{\varepsilon} \partial_{\alpha}\left(U^{\varepsilon} \hat{\mathbf{n}}^{\varepsilon}+V^{\varepsilon} \hat{\mathbf{t}}^{\varepsilon}\right.}{\left(z_{\alpha}^{\varepsilon}\right)}\right)\right\} .
\end{aligned}
$$

For $R_{2}$, we rewrite (26) by substituting for $z_{t}$ :

$$
\begin{aligned}
R_{2}=-\operatorname{Re}\left\{\frac{z_{\alpha}}{2 i}[H, U \hat{\mathbf{n}}+V \hat{\mathbf{t}}]\left(\frac{1}{z_{\alpha}}\left(\frac{\gamma}{z_{\alpha}}\right)_{\alpha}\right)\right\}+\operatorname{Re}\left\{z_{\alpha}(U \hat{\mathbf{n}}+V \hat{\mathbf{t}}) \mathcal{K}\left[z_{d}\right]\left(\left(\frac{\gamma}{z_{\alpha}}\right)_{\alpha}\right)\right\} & -\operatorname{Re}\left\{z_{\alpha} \mathcal{K}\left[z_{d}\right]\left((U \hat{\mathbf{n}}+V \hat{\mathbf{t}})\left(\frac{\gamma}{z_{\alpha}}\right)_{\alpha}\right)\right\} .
\end{aligned}
$$

Defining $R_{2}^{\varepsilon}$ is then straightforward:

$$
\begin{aligned}
R_{2}^{\varepsilon}=-\operatorname{Re}\left\{\frac{z_{\alpha}^{\varepsilon}}{2 i}\left[H, U^{\varepsilon} \hat{\mathbf{n}}^{\varepsilon}+V^{\varepsilon} \hat{\mathbf{t}}^{\varepsilon}\right]\left(\frac{1}{z_{\alpha}^{\varepsilon}}\left(\frac{\gamma^{\varepsilon}}{z_{\alpha}^{\varepsilon}}\right)_{\alpha}\right)\right\}+ & \operatorname{Re}\left\{z_{\alpha}^{\varepsilon}\left(U^{\varepsilon} \hat{\mathbf{n}}^{\varepsilon}+V^{\varepsilon} \hat{\mathbf{t}}^{\varepsilon}\right) \mathcal{K}\left[z_{d}^{\varepsilon}\right]\left(\left(\frac{\gamma^{\varepsilon}}{z_{\alpha}^{\varepsilon}}\right)\right)\right\} \\
& -\operatorname{Re}\left\{z_{\alpha}^{\varepsilon} \mathcal{K}\left[z_{d}^{\varepsilon}\right]\left(\left(U^{\varepsilon} \hat{\mathbf{n}}^{\varepsilon}+V^{\varepsilon} \hat{\mathbf{t}}^{\varepsilon}\right)\left(\frac{\gamma^{\varepsilon}}{z_{\alpha}^{\varepsilon}}\right)\right)\right\} .
\end{aligned}
$$

\subsection{Auxiliary estimates}

In this section, we give estimates for quantities like $\mathbf{W}^{\varepsilon}$ or $V_{W}^{\varepsilon}$, in terms of norms of $\theta^{\varepsilon}$ and $\gamma^{\varepsilon}$. We note that the same estimates apply when there is no regularization, i.e., when $\varepsilon=0$, and the proof is exactly the same. The estimates on regularized quantitites is used in the proof of Theorem 10, while those for unregularized quantities is used in the proof of Theorem 12.

Lemma 7 Let $\left(\theta^{\varepsilon}, \gamma^{\varepsilon}\right) \in \mathcal{O}$ be given, such that $\theta^{\varepsilon}$ satisfies $\left\langle\left\langle\sin \left(\theta^{\varepsilon}\right)\right\rangle=0\right.$. Then, the following estimates are satisfied:

$$
\begin{gathered}
\left\|z_{\alpha}^{\varepsilon}\right\|_{H^{s}} \leq c\left(1+\left\|\theta^{\varepsilon}\right\|_{H^{s}}\right), \\
\left\|z_{d}^{\varepsilon}\right\|_{H^{s+1}} \leq c\left(1+\left\|\theta^{\varepsilon}\right\|_{H^{s}}\right), \\
\left\|\hat{\mathbf{t}}^{\varepsilon}\right\|_{H^{s}} \leq c\left(1+\left\|\theta^{\varepsilon}\right\|_{H^{s}}\right) \\
\left\|\hat{\mathbf{n}}^{\varepsilon}\right\|_{H^{s}} \leq c\left(1+\left\|\theta^{\varepsilon}\right\|_{H^{s}}\right) \\
\left\|\mathbf{m}^{\varepsilon}\right\|_{H^{s}} \leq c_{1}\left\|\gamma^{\varepsilon}\right\|_{H^{s-3 / 2}} \exp \left\{c_{2}\left\|\theta^{\varepsilon}\right\|_{H^{s}}\right\}, \\
\left\|U^{\varepsilon}\right\|_{H^{s-3 / 2}} \leq c_{1}\left\|\gamma^{\varepsilon}\right\|_{H^{s-3 / 2}} \exp \left\{c_{2}\left\|\theta^{\varepsilon}\right\|_{H^{s}}\right\}, \\
\left\|\mathbf{W}^{\varepsilon} \cdot \hat{\mathbf{t}}^{\varepsilon}\right\|_{H^{s-1 / 2}} \leq c_{1}\left\|\gamma^{\varepsilon}\right\|_{H^{s-3 / 2}} \exp \left\{c_{2}\left\|\theta^{\varepsilon}\right\|_{H^{s}}\right\} \\
\left\|V_{W}^{\varepsilon}\right\|_{H^{s-1 / 2}} \leq c_{1}\left\|\gamma^{\varepsilon}\right\|_{H^{s-3 / 2}} \exp \left\{c_{2}\left\|\theta^{\varepsilon}\right\|_{H^{s}}\right\}, \\
\left\|V^{\varepsilon}\right\|_{H^{s-1 / 2}} \leq c_{1}\left\|\gamma^{\varepsilon}\right\|_{H^{s-3 / 2}} \exp \left\{c_{2}\left\|\theta^{\varepsilon}\right\|_{H^{s}}\right\} \\
\left\|Q_{1}^{\varepsilon}\right\|_{H^{s-1}} \leq c\left(1+\left\|\theta^{\varepsilon}\right\|_{H^{s}}\right),
\end{gathered}
$$




$$
\begin{gathered}
\left\|Q_{2}^{\varepsilon}\right\|_{H^{s-3 / 2}} \leq c_{1}\left\|\gamma^{\varepsilon}\right\|_{H^{s-3 / 2}} \exp \left\{c_{2}\left\|\theta^{\varepsilon}\right\|_{H^{s}}\right\}, \\
\left\|Q_{3}^{\varepsilon}\right\|_{H^{s-3 / 2}} \leq c_{1}\left(\left\|\gamma^{\varepsilon}\right\|_{H^{s-3 / 2}}^{2}+1\right) \exp \left\{c_{2}\left\|\theta^{\varepsilon}\right\|_{H^{s}}\right\}, \\
\left|\mu^{\varepsilon}\right| \leq c_{1}\left\|\gamma^{\varepsilon}\right\|_{H^{s-3 / 2}} \exp \left\{c_{2}\left\|\theta^{\varepsilon}\right\|_{H^{s}}\right\}
\end{gathered}
$$

where the constants are independent of $\varepsilon$.

Proof: The estimate (54) follows immediately from (37) and (38), together with (a) a standard composition estimate [37], and (b) the fact that the definition of $\mathcal{O}$ includes a bound on the length. The estimates (56) and (57), in light of (40), are similar. Since $z_{d}^{\varepsilon}$ is defined in (39) by integrating $x_{\alpha}^{\varepsilon}$ and $y_{\alpha}^{\varepsilon}$, the estimate (55) follows.

To establish (58), we use Lemma 2 to bound the first term on the right-hand side of (47), and we use Lemma 5 to bound the second term on the right-hand side of (47). To establish (58), we also rely on (54) and (55). The estimate (59) follows from (48) and the bound (58) on $\mathbf{m}^{\varepsilon}$. To establish the estimate on $\mathbf{W}^{\varepsilon} \cdot \hat{\mathbf{t}}^{\varepsilon}$, we use $\left(\mathbf{W}^{\varepsilon} \cdot \hat{\mathbf{t}}^{\varepsilon}\right)_{\alpha}=\mathbf{W}_{\alpha}^{\varepsilon} \cdot \hat{\mathbf{t}}^{\varepsilon}+U^{\varepsilon} \theta_{\alpha}^{\varepsilon}$; the estimate then follows by taking the tangential component of (48) and using the previous estimate on $U^{\varepsilon}$. The estimates on $V_{W}^{\varepsilon}$ and $V^{\varepsilon}$ readily follow from (45) and (49) and the bound on $\mathbf{m}^{\varepsilon}$. The estimates of $Q_{1}^{\varepsilon}$ and $Q_{2}^{\varepsilon}$ are easily obtained from their definitions (46) and (50) using prior estimates.

The estimate on $Q_{3}^{\varepsilon}$ defined in (51) is the most involved. We first obtain bounds on $R_{2}^{\varepsilon}$ and $R_{3}^{\varepsilon}$ of the form $\left\|R_{i}^{\varepsilon}\right\|_{H^{s-3 / 2}} \leq c_{1}\left\|\gamma^{\varepsilon}\right\|_{H^{s-3 / 2}} \exp \left\{c_{2}\left\|\theta^{\varepsilon}\right\|_{H^{s}}\right\}$ for $i=2$, 3. The estimate on $R_{2}^{\varepsilon}$ follows from using Lemma 5 to bound the first term on the right hand side of (53) and Lemma 2 to bound the second and third terms. The estimate on $R_{3}^{\varepsilon}$ uses Lemma 4 to bound the first term on the right hand side of (52) and Lemma 2 to bound the fourth term; estimates on the second and third terms are straighforward, with the Schwartz inequality used to obtain a bound on the integral quantity $\int_{0}^{2 \pi} \theta_{\alpha} U d \alpha$. Proceeding with the estimate on $Q_{3}^{\varepsilon}$, we use previous estimates to obtain a bound on the first term in (51):

$$
\left\|\tilde{Q}_{3}^{\varepsilon}\right\|_{H^{s-3 / 2}} \leq c_{1}\left(\left\|\gamma^{\varepsilon}\right\|_{H^{s-3 / 2}}^{2} \exp \left\{c_{2}\left\|\theta^{\varepsilon}\right\|_{H^{s}}\right\}+\left\|\theta^{\varepsilon}\right\|_{H^{s}}\right) .
$$

Next, since we have assumed $s$ is sufficiently large so that $\Xi^{\varepsilon} \in H^{0}$, Lemma 6 implies that the second term in (51) satisfies $\left(I+2 A \mathcal{J}\left[z_{d}^{\varepsilon}\right]\right)^{-1} \Xi^{\varepsilon}=\gamma_{t}^{\varepsilon} \in H^{0}$, and in particular $\left\|\gamma_{t}^{\varepsilon}\right\|_{0} \leq c$. We then find an estimate on the second term in (51), $\mathcal{J}\left[z_{d}^{\varepsilon}\right] \gamma_{t}^{\varepsilon}$, from $H_{0}$ to the higher norm $H_{s-1}$. To obtain this estimate, we write

$$
\begin{aligned}
\mathcal{J}\left[z_{d}^{\varepsilon}\right] \gamma_{t}^{\varepsilon} & =\operatorname{Re}\left(\frac{i z_{\alpha}^{\varepsilon}}{4 \pi} \mathrm{PV} \int_{0}^{2 \pi} \gamma_{t}^{\varepsilon \prime} \cot \frac{z_{d}^{\varepsilon}-z_{d}^{\varepsilon \prime}}{2} d \alpha^{\prime}\right) \\
& =\operatorname{Re}\left(-z_{\alpha}^{\varepsilon} \mathcal{K}\left[z_{d}^{\varepsilon}\right] \gamma_{t}^{\varepsilon}+i z_{\alpha}^{\varepsilon}\left[H, \frac{1}{z_{\alpha}^{\varepsilon}}\right]\left(\gamma_{t}^{\varepsilon}\right)\right)
\end{aligned}
$$

and then apply Lemmas 2 and 4 to get

$$
\begin{aligned}
\left\|\mathcal{J}\left[z_{d}^{\varepsilon}\right] \gamma_{t}^{\varepsilon}\right\|_{H_{s-1}} & \leq c\left\|\gamma_{t}^{\varepsilon}\right\|_{H_{0}} \exp \left\{c\left\|\theta^{\varepsilon}\right\|_{H_{s}}\right\} \\
& \leq c_{1} \exp \left\{c\left\|\theta^{\varepsilon}\right\|_{H_{s}}\right\}
\end{aligned}
$$

Combining (67) with (68) gives the estimate (65). Finally, the estimate on $\mu^{\varepsilon}$ is readily obtained from its definition (42) using previous estimates and the Schwartz inequality. 


\subsection{The energy estimate}

We state the Picard theorem for ordinary differential equations on a Banach space; the particular statement we quote is from [31], and a similar statement can be found in [44].

Theorem 8 (Picard) Let $O \subseteq B$ be an open subset of a Banach space, B. Let $F: O \rightarrow B$. Assume that $F$ is locally Lipschitz continuous, i.e., that for all $x \in O$, there exists an open neighborhood of $x, U_{x} \subseteq O$, and $c>0$ such that for all $x_{1}, x_{2} \in U_{x}$,

$$
\left\|F\left(x_{1}\right)-F\left(x_{2}\right)\right\|_{B} \leq c\left\|x_{1}-x_{2}\right\|_{B}
$$

Then, for every $x_{0} \in O$, there exists $T>0$ and $x \in C^{1}((-T, T) ; O)$ such that $x$ is the solution of the initial value problem

$$
\frac{d x}{d t}=F(x), \quad x(0)=x_{0} .
$$

In order to use the Picard theorem, we introduce the following open set, $\mathcal{O}$. Let positive constants $\bar{d}_{1}, \bar{d}_{2}$, and $\bar{d}_{3}$ be given. We let $\mathcal{O}$ be the subset of $H^{s} \times H^{s-3 / 2}$ such that for all $\left(f_{1}, f_{2}\right) \in \mathcal{O}$, the following three conditions are satisfied:

$$
\begin{gathered}
\left\|\left(f_{1}, f_{2}\right)\right\|_{H^{s} \times H^{s-3 / 2}}<\bar{d}_{1}, \quad L\left[f_{1}\right]<\bar{d}_{2}, \\
\left|q_{1}\left[f_{1}\right]\left(\alpha, \alpha^{\prime}\right)\right|>\bar{d}_{3}, \quad \forall \alpha, \alpha^{\prime} .
\end{gathered}
$$

The conditions in (69) state that the set $\mathcal{O}$ is a subset of an open ball, such that the curves generated by tangent angle $f_{1}$ all have bounded length. The condition in (70) states that the curves generated by tangent angle $f_{1}$ are not close to self-intersection.

Theorem 9 Let $\left(\theta_{0}, \gamma_{0}\right) \in \mathcal{O}$ be given, with $\theta_{0}$ satisfying $\left\langle\sin \left(\theta_{0}\right)\right\rangle=0$. There exists $T_{\varepsilon}>0$ and $\left(\theta^{\varepsilon}, \gamma^{\varepsilon}\right) \in C^{1}\left(\left(-T^{\varepsilon}, T^{\varepsilon}\right) ; \mathcal{O}\right)$ such that $\left(\theta^{\varepsilon}, \gamma^{\varepsilon}\right)$ is the unique solution of the initial value problem given by (41) with initial data $\left(\theta_{0}, \gamma_{0}\right)$.

We have thus demonstrated the existence of solutions to the mollified system. We would like to pass to the limit as $\varepsilon \rightarrow 0^{+}$. However, we cannot do this yet, as the time interval from Theorem 9 could go to zero as $\varepsilon$ vanishes. Our next step is to prove an energy estimate, uniformly in $\varepsilon$, for the solutions $\left(\theta^{\varepsilon}, \gamma^{\varepsilon}\right)$. We can then use the continuation theorem for ordinary differential equations on a Banach space to find that the solutions of the mollified system exist on a common time interval; after this, we will be able to pass to the limit as the regularization vanishes.

Theorem 10 Let $\left(\theta_{0}, \gamma_{0}\right) \in \mathcal{O}$ be given, with $\theta_{0}$ satisfying $\left\langle\sin \left(\theta_{0}\right)\right\rangle=0$. Let $\varepsilon>0$ be given. Let $\left(\theta^{\varepsilon}, \gamma^{\varepsilon}\right) \in C([0, T] ; \mathcal{O})$ be a solution of $(41)$, with initial conditions $\left(\theta_{0}, \gamma_{0}\right)$. (Note that this $T$ may depend on $\varepsilon$.) Then there exist constants $c_{1} \in(0, \infty), c_{2} \in(0,1)$, and $c_{3} \in(0, \infty)$, depending only on $s, \bar{d}_{1}, \bar{d}_{2}, \bar{d}_{3},\left\|\theta_{0}\right\|_{H^{s}}$, and $\left\|\gamma_{0}\right\|_{H^{s-3 / 2}}$, such that

$$
\left\|\theta^{\varepsilon}\right\|_{H^{s}}^{2}+\left\|\gamma^{\varepsilon}\right\|_{H^{s-3 / 2}}^{2} \leq-c_{1} \ln \left(c_{2}-c_{3} t\right) .
$$

Proof: We will define an energy functional, $E$, such that

$$
\frac{1}{2}\left(\left\|\theta^{\varepsilon}\right\|_{s}^{2}+\left\|\gamma^{\varepsilon}\right\|_{s-3 / 2}^{2}\right) \leq E,
$$


and such that there exist constants $C_{1}>0$ and $C_{2}>0$ such that

$$
\frac{d E}{d t} \leq C_{1} \exp \left\{C_{2} E\right\}
$$

The inequality (73) implies

$$
E(t) \leq-\frac{-\ln \left(e^{-C_{2} E(0)}-C_{1} C_{2} t\right)}{C_{2}} .
$$

In light of (72), and renaming the constants, we see that (71) is then satisfied. Thus, we need only to establish (73). Throughout the proof, we make frequent use of the properties of the mollifier of $\Xi_{\varepsilon}$, i.e., that it is self-adjoint and commutes with derivatives.

As in the example of Section 2, we will define the energy a bit at a time. The energy will be given by

$$
E=E_{0}+E_{1}+E_{2}+E_{3}+E_{4}+E_{5}
$$

where we will now give the definition of $E_{0}, E_{1}$, and $E_{2}$. The rest of the terms will be defined as needed. We have

$$
\begin{gathered}
E_{0}=\frac{1}{2} \int_{0}^{2 \pi}\left(\theta^{\varepsilon}\right)^{2}+\left(\gamma^{\varepsilon}\right)^{2} d \alpha \\
E_{1}=\frac{c_{1}(t)}{2} \int_{0}^{2 \pi}\left(\partial_{\alpha}^{s} \theta^{\varepsilon}\right)^{2} d \alpha \\
E_{2}=\frac{c_{2}(t)}{2} \int_{0}^{2 \pi}\left(\partial_{\alpha}^{s-2} \gamma^{\varepsilon}\right) \Lambda\left(\partial_{\alpha}^{s-2} \gamma^{\varepsilon}\right) d \alpha
\end{gathered}
$$

We see that property (72) is satisfied as long as $c_{1}(t) \geq 1, c_{2}(t) \geq 1$, for all $t$, and $E_{i} \geq 0$ for $i \in\{3,4,5\}$. When we choose $c_{1}, c_{2}, E_{3}, E_{4}$, and $E_{5}$, all of these properties will be satisfied.

To begin, we take the time derivative of $E_{0}$ :

$$
\frac{d E_{0}}{d t}=\int_{0}^{2 \pi} \theta^{\varepsilon} \theta_{t}^{\varepsilon}+\gamma^{\varepsilon} \gamma_{t}^{\varepsilon} d \alpha
$$

Since $s$ is sufficiently large, it is immediate, from the evolution equation (41), the definitions (42), (43), (44), and related equations, as well as the estimates of Section 5.2, that this can be bounded in terms of the energy:

$$
\frac{d E_{0}}{d t} \leq C_{1} \exp \left\{C_{2} E\right\}
$$

We next take the time derivative of $E_{1}$ :

$$
\frac{d E_{1}}{d t}=\frac{d c_{1}}{d t} \cdot \frac{1}{2} \int_{0}^{2 \pi}\left(\partial_{\alpha}^{s} \theta^{\varepsilon}\right)^{2} d \alpha+c_{1} \int_{0}^{2 \pi}\left(\partial_{\alpha}^{s} \theta^{\varepsilon}\right)\left(\partial_{\alpha}^{s} \theta_{t}^{\varepsilon}\right) d \alpha .
$$

We note that $\partial_{\alpha} \mu^{\varepsilon}=0$, so there is no contribution from $\mu^{\varepsilon}$ in (75). To proceed with (75), we will write a formula for $\partial_{\alpha}^{s} \theta_{t}^{\varepsilon}$. Applying $\partial_{\alpha}^{s}$ to (43), we get

$$
\partial_{\alpha}^{s} \theta_{t}^{\varepsilon}=\frac{2 \pi^{2}}{\left(L^{\varepsilon}\right)^{2}} \chi_{\varepsilon} H\left(\partial_{\alpha}^{s+1} \gamma^{\varepsilon}\right)+\frac{2 \pi}{L^{\varepsilon}}\left(\chi_{\varepsilon}\left(\partial_{\alpha}^{s} V_{W}^{\varepsilon}\right)\left(\chi_{\varepsilon} \theta_{\alpha}^{\varepsilon}\right)\right)+\frac{2 \pi}{L^{\varepsilon}} \chi_{\varepsilon}\left(V_{W}^{\varepsilon}\left(\chi_{\varepsilon} \partial_{\alpha}^{s+1} \theta^{\varepsilon}\right)\right)+\Phi_{1},
$$


where $\Phi_{1}$ is given by the formula

$$
\Phi_{1}=\frac{2 \pi}{L^{\varepsilon}} \partial_{\alpha}^{s}\left(\mathbf{m}^{\varepsilon} \cdot \hat{\mathbf{n}}^{\varepsilon}\right)+\frac{2 \pi}{L^{\varepsilon}} \chi_{\varepsilon}\left(\sum_{j=1}^{s-1}\left(\begin{array}{l}
s \\
j
\end{array}\right)\left(\partial_{\alpha}^{j} V_{W}^{\varepsilon}\right)\left(\chi_{\varepsilon} \partial_{\alpha}^{s-j+1} \theta^{\varepsilon}\right)\right) .
$$

All of the summands here involve at most $s-1$ derivatives of $V_{W}^{\varepsilon}$, and at most $s$ derivatives of $\theta^{\varepsilon}$. Therefore, the estimates of Section 5.2 and (72) immediately imply that $\left\|\Phi_{1}\right\|_{0} \leq C_{1} \exp \left\{C_{2} E\right\}$.

We then plug (76) into (75). Using the property that the mollifier $\chi_{\varepsilon}$ is self-adjoint and commutes with derivatives, we compute the following:

$$
\begin{aligned}
\frac{d E_{1}}{d t} & =\frac{2 \pi^{2} c_{1}}{\left(L^{\varepsilon}\right)^{2}} \int_{0}^{2 \pi}\left(\chi_{\varepsilon} \partial_{\alpha}^{s} \theta^{\varepsilon}\right)\left(\chi_{\varepsilon} H \partial_{\alpha}^{s+1} \gamma^{\varepsilon}\right) d \alpha+\frac{2 \pi c_{1}}{L^{\varepsilon}} \int_{0}^{2 \pi}\left(\chi_{\varepsilon} \partial_{\alpha}^{s} \theta^{\varepsilon}\right)\left(\partial_{\alpha}^{s} V_{W}^{\varepsilon}\right)\left(\chi_{\varepsilon} \theta_{\alpha}^{\varepsilon}\right) d \alpha \\
& +\frac{2 \pi c_{1}}{L^{\varepsilon}} \int_{0}^{2 \pi}\left(\chi_{\varepsilon} \partial_{\alpha}^{s} \theta^{\varepsilon}\right)\left(\chi_{\varepsilon} \partial_{\alpha}^{s+1} \theta^{\varepsilon}\right) V_{W}^{\varepsilon} d \alpha+\frac{d c_{1}}{d t} \cdot \frac{1}{2} \int_{0}^{2 \pi}\left(\partial_{\alpha}^{s} \theta^{\varepsilon}\right)^{2} d \alpha+\int_{0}^{2 \pi}\left(\partial_{\alpha}^{s} \theta^{\varepsilon}\right) \Phi_{1} d \alpha .
\end{aligned}
$$

For the third term on the right-hand side of (77), we recognize a perfect derivative and integrate by parts:

$$
\frac{d E_{1}}{d t}=\frac{2 \pi^{2} c_{1}}{\left(L^{\varepsilon}\right)^{2}} \int_{0}^{2 \pi}\left(\chi_{\varepsilon} \partial_{\alpha}^{s} \theta^{\varepsilon}\right)\left(\chi_{\varepsilon} H \partial_{\alpha}^{s+1} \gamma^{\varepsilon}\right) d \alpha+\frac{2 \pi c_{1}}{L^{\varepsilon}} \int_{0}^{2 \pi}\left(\chi_{\varepsilon} \partial_{\alpha}^{s} \theta^{\varepsilon}\right)\left(\partial_{\alpha}^{s} V_{W}^{\varepsilon}\right)\left(\chi_{\varepsilon} \theta_{\alpha}^{\varepsilon}\right) d \alpha+\Psi_{1},
$$

where $\Psi_{1}$ is defined as

$$
\Psi_{1}=-\frac{\pi c_{1}}{L^{\varepsilon}} \int_{0}^{2 \pi}\left(\chi_{\varepsilon} \partial_{\alpha}^{s} \theta^{\varepsilon}\right)^{2} \partial_{\alpha} V_{W}^{\varepsilon} d \alpha+\frac{d c_{1}}{d t} \cdot \frac{1}{2} \int_{0}^{2 \pi}\left(\partial_{\alpha}^{s} \theta^{\varepsilon}\right)^{2} d \alpha+c_{1} \int_{0}^{2 \pi}\left(\partial_{\alpha}^{s} \theta^{\varepsilon}\right) \Phi_{1} d \alpha .
$$

Before we are able to choose $c_{1}$, we must compute $\frac{d E_{2}}{d t}$, which we now do. To begin, we have simply

$$
\frac{d E_{2}}{d t}=\frac{d c_{2}}{d t} \cdot \frac{1}{2} \int_{0}^{2 \pi}\left(\partial_{\alpha}^{s-2} \gamma^{\varepsilon}\right) \Lambda\left(\partial_{\alpha}^{s-2} \gamma^{\varepsilon}\right) d \alpha+c_{2} \int_{0}^{2 \pi}\left(\partial_{\alpha}^{s-2} \gamma_{t}^{\varepsilon}\right)\left(H \partial_{\alpha}^{s-1} \gamma^{\varepsilon}\right) d \alpha
$$

We next must compute $\partial_{\alpha}^{s-2} \gamma_{t}^{\varepsilon}$ :

$$
\partial_{\alpha}^{s-2} \gamma_{t}^{\varepsilon}=-\widetilde{S}^{\varepsilon} \chi_{\varepsilon} \partial_{\alpha}^{s+2} \theta^{\varepsilon}-\widetilde{S}^{\varepsilon} \chi_{\varepsilon}\left(\left(\chi_{\varepsilon} \partial_{\alpha}^{s} \theta^{\varepsilon}\right) Q_{1}^{\varepsilon}\right)+\chi_{\varepsilon}\left(\left(\chi_{\varepsilon} \partial_{\alpha}^{s-1} \gamma^{\varepsilon}\right) Q_{2}^{\varepsilon}\right)+\Phi_{2},
$$

where $\Phi_{2}$ is defined as

$$
\Phi_{2}=-\sum_{j=0}^{s-3}\left(\begin{array}{c}
s-2 \\
j
\end{array}\right) \widetilde{S}^{\varepsilon} \chi_{\varepsilon}\left(\left(\partial_{\alpha}^{j+2} \theta^{\varepsilon}\right)\left(\partial_{\alpha}^{s-2-j} Q_{1}^{\varepsilon}\right)\right)+\sum_{j=0}^{s-3}\left(\begin{array}{c}
s-2 \\
j
\end{array}\right) \chi_{\varepsilon}\left(\left(\chi_{\varepsilon} \partial_{\alpha}^{j+1} \gamma^{\varepsilon}\right)\left(\partial_{\alpha}^{s-2-j} Q_{2}^{\varepsilon}\right)\right)+\partial_{\alpha}^{s-2} Q_{3}^{\varepsilon} .
$$

From the estimates of Section 5.2, since the summands here involve at most $s-1$ derivatives of $\theta^{\varepsilon}$, at most $s-2$ derivatives of $\gamma^{\varepsilon}$, and at most $s-2$ derivatives of $Q_{i}$ for $i \in\{1,2,3\}$, the estimates of Section 5.2 imply $\left\|\Phi_{2}\right\|_{1 / 2} \leq C_{1} \exp \left\{C_{2} E\right\}$.

We plug (81) into (80), and we again repeatedly use the fact that $\chi_{\varepsilon}$ is self-adjoint (as well as the fact that $\left.\chi_{\varepsilon}=\chi_{\varepsilon}^{2}\right)$ :

$$
\begin{gathered}
\frac{d E_{2}}{d t}=-c_{2} \widetilde{S}^{\varepsilon} \int_{0}^{2 \pi}\left(\chi_{\varepsilon} H \partial_{\alpha}^{s-1} \gamma^{\varepsilon}\right)\left(\chi_{\varepsilon} \partial_{\alpha}^{s+2} \theta^{\varepsilon}\right) d \alpha-c_{2} \widetilde{S}^{\varepsilon} \int_{0}^{2 \pi}\left(\chi_{\varepsilon} H \partial_{\alpha}^{s-1} \gamma^{\varepsilon}\right)\left(\chi_{\varepsilon} \partial_{\alpha}^{s} \theta^{\varepsilon}\right) Q_{1}^{\varepsilon} d \alpha \\
+c_{2} \int_{0}^{2 \pi}\left(\chi_{\varepsilon} H \partial_{\alpha}^{s-1} \gamma^{\varepsilon}\right)\left(\chi_{\varepsilon} \partial_{\alpha}^{s-1} \gamma^{\varepsilon}\right) Q_{2}^{\varepsilon} d \alpha+\frac{d c_{2}}{d t} \cdot \frac{1}{2} \int_{0}^{2 \pi}\left(\partial_{\alpha}^{s-2} \gamma^{\varepsilon}\right) \Lambda\left(\partial_{\alpha}^{s-2} \gamma^{\varepsilon}\right) d \alpha+c_{2} \int_{0}^{2 \pi}\left(\Lambda \partial_{\alpha}^{s-2} \gamma^{\varepsilon}\right) \Phi_{2} d \alpha .
\end{gathered}
$$


We integrate the first term on the right-hand side of (82) by parts twice, and we recall the definition of $\widetilde{S}^{\varepsilon}$ :

$$
\frac{d E_{2}}{d t}=-\frac{8 \pi^{3} S c_{2}}{\left(L^{\varepsilon}\right)^{3}} \int_{0}^{2 \pi}\left(\chi_{\varepsilon} H \partial_{\alpha}^{s+1} \gamma^{\varepsilon}\right)\left(\chi_{\varepsilon} \partial_{\alpha}^{s} \theta^{\varepsilon}\right) d \alpha+c_{2} \widetilde{S}^{\varepsilon} \int_{0}^{2 \pi}\left(\chi_{\varepsilon} H \partial_{\alpha}^{s-1} \gamma^{\varepsilon}\right)\left(\chi_{\varepsilon} \partial_{\alpha}^{s} \theta^{\varepsilon}\right) Q_{1}^{\varepsilon} d \alpha+\Psi_{2},
$$

where $\Psi_{2}$ is defined as

$$
\begin{aligned}
\Psi_{2}=c_{2} \int_{0}^{2 \pi}\left(\chi_{\varepsilon} H \partial_{\alpha}^{s-1} \gamma^{\varepsilon}\right)\left(\chi_{\varepsilon} \partial_{\alpha}^{s-1} \gamma^{\varepsilon}\right) Q_{2}^{\varepsilon} d \alpha+\frac{d c_{2}}{d t} \cdot \frac{1}{2} \int_{0}^{2 \pi}\left(\partial_{\alpha}^{s-2} \gamma^{\varepsilon}\right) \Lambda\left(\partial_{\alpha}^{s-2} \gamma^{\varepsilon}\right) d \alpha \\
\\
+c_{2} \int_{0}^{2 \pi}\left(\Lambda \partial_{\alpha}^{s-2} \gamma^{\varepsilon}\right) \Phi_{2} d \alpha
\end{aligned}
$$

We now add (78) and (83), and group the like terms:

$$
\begin{aligned}
& \frac{d E_{1}}{d t}+\frac{d E_{2}}{d t}=\left[\frac{2 \pi^{2} c_{1}}{\left(L^{\varepsilon}\right)^{2}}-\frac{8 \pi^{3} S c_{2}}{\left(L^{\varepsilon}\right)^{3}}\right] \int_{0}^{2 \pi}\left(\chi_{\varepsilon} H \partial_{\alpha}^{s+1} \gamma^{\varepsilon}\right)\left(\chi_{\varepsilon} \partial_{\alpha}^{s} \theta^{\varepsilon}\right) d \alpha \\
& +\frac{2 \pi c_{1}}{L^{\varepsilon}} \int_{0}^{2 \pi}\left(\chi_{\varepsilon} \partial_{\alpha}^{s} \theta^{\varepsilon}\right)\left(\partial_{\alpha}^{s} V_{W}^{\varepsilon}\right)\left(\chi_{\varepsilon} \theta_{\alpha}^{\varepsilon}\right) d \alpha-c_{2} \widetilde{S}^{\varepsilon} \int_{0}^{2 \pi}\left(\chi_{\varepsilon} H \partial_{\alpha}^{s-1} \gamma^{\varepsilon}\right)\left(\chi_{\varepsilon} \partial_{\alpha}^{s} \theta^{\varepsilon}\right) Q_{1}^{\varepsilon} d \alpha+\Psi_{1}+\Psi_{2} .
\end{aligned}
$$

To make the first term on the right-hand side of (85) vanish, we choose $c_{1}$ and $c_{2}$ to satisfy

$$
\frac{2 \pi^{2} c_{1}}{\left(L^{\varepsilon}\right)^{2}}-\frac{8 \pi^{3} S c_{2}}{\left(L^{\varepsilon}\right)^{3}}=0
$$

this is satisfied as long as

$$
\frac{c_{1}}{c_{2}}=\frac{4 \pi S}{L^{\varepsilon}}
$$

Recall also the previous conditions $c_{1} \geq 1$ and $c_{2} \geq 1$. Therefore, we choose

$$
c_{2}=\max \left\{L^{\varepsilon}, \frac{L^{\varepsilon}}{S}\right\}, \quad c_{1}=\frac{4 \pi S c_{2}}{L^{\varepsilon}} .
$$

Clearly $c_{2} \geq 1$ by definition; for $c_{1}$, it follows that $c_{1}(t) \geq 1$ for all $t$ since the horizontal periodicity of the interface implies $L^{\varepsilon}(t) \geq 2 \pi$ for all $t$. Having made these choices for $c_{1}$ and $c_{2}$, we have

$\frac{d E_{1}}{d t}+\frac{d E_{2}}{d t}=\frac{2 \pi c_{1}}{L^{\varepsilon}} \int_{0}^{2 \pi}\left(\chi_{\varepsilon} \partial_{\alpha}^{s} \theta^{\varepsilon}\right)\left(\partial_{\alpha}^{s} V_{W}^{\varepsilon}\right)\left(\chi_{\varepsilon} \theta_{\alpha}^{\varepsilon}\right) d \alpha-c_{2} \widetilde{S}^{\varepsilon} \int_{0}^{2 \pi}\left(\chi_{\varepsilon} H \partial_{\alpha}^{s-1} \gamma^{\varepsilon}\right)\left(\chi_{\varepsilon} \partial_{\alpha}^{s} \theta^{\varepsilon}\right) Q_{1}^{\varepsilon} d \alpha+\Psi_{1}+\Psi_{2}$.

The integrals on the right-hand side of (87) cannot be estimated in terms of the energy; we must choose $E_{3}$ to achieve an additional cancellation. First, however, we will rewrite (87) in order to more fully understand the terms which cannot be bounded in terms of the energy.

Applying $\partial_{\alpha}^{s}$ to $V_{W}^{\varepsilon}$, using (45), and extracting the leading-order term, we have

$$
\begin{aligned}
\partial_{\alpha}^{s} V_{W}^{\varepsilon}=\frac{\pi}{L^{\varepsilon}}\left(\chi_{\varepsilon} \theta_{\alpha}^{\varepsilon}\right) H\left(\chi_{\varepsilon} \partial_{\alpha}^{s-1} \gamma^{\varepsilon}\right)+ & \frac{\pi}{L^{\varepsilon}}\left[H, \chi_{\varepsilon} \theta_{\alpha}^{\varepsilon}\right]\left(\chi_{\varepsilon} \partial_{\alpha}^{s-1} \gamma^{\varepsilon}\right) \\
& +\frac{\pi}{L^{\varepsilon}} \sum_{j=0}^{s-2}\left(\begin{array}{c}
s-1 \\
j
\end{array}\right) H\left(\left(\chi_{\varepsilon} \partial_{\alpha}^{j} \gamma^{\varepsilon}\right)\left(\chi_{\varepsilon} \partial_{\alpha}^{s-j} \theta^{\varepsilon}\right)\right)-\partial_{\alpha}^{s}\left(\mathbf{m}^{\varepsilon} \cdot \hat{\mathbf{t}}^{\varepsilon}\right) .
\end{aligned}
$$


We substitute (88) and (46) into (87), and we also use (86) to substitute for $c_{1}$. We collect like terms, and we collect lower-order terms, arriving at the following:

$$
\frac{d E_{1}}{d t}+\frac{d E_{2}}{d t}=c_{2} \int_{0}^{2 \pi}\left[\frac{2 \pi S \bar{c}_{1}}{L^{\varepsilon}}-\frac{4 \pi^{3} S}{\left(L^{\varepsilon}\right)^{3}}\left(\chi_{\varepsilon} \theta_{\alpha}^{\varepsilon}\right)^{2}\right]\left(\chi_{\varepsilon} \partial_{\alpha}^{s} \theta^{\varepsilon}\right)\left(\chi_{\varepsilon} H \partial_{\alpha}^{s-1} \gamma^{\varepsilon}\right) d \alpha+\Psi_{3},
$$

where $\Psi_{3}$ is given by

$$
\begin{gathered}
\Psi_{3}=\Psi_{1}+\Psi_{2}+\frac{8 \pi^{3} S c_{2}}{\left(L^{\varepsilon}\right)^{3}} \int_{0}^{2 \pi}\left(\chi_{\varepsilon} \partial_{\alpha}^{s} \theta^{\varepsilon}\right)\left(\chi_{\varepsilon} \theta_{\alpha}^{\varepsilon}\right)\left(\sum_{j=0}^{s-2}\left(\begin{array}{c}
s-1 \\
j
\end{array}\right) H\left(\left(\chi_{\varepsilon} \partial_{\alpha}^{j} \gamma^{\varepsilon}\right)\left(\chi_{\varepsilon} \partial_{\alpha}^{s-j} \theta^{\varepsilon}\right)\right)\right) d \alpha \\
+\frac{8 \pi^{3} S c_{2}}{\left(L^{\varepsilon}\right)^{3}} \int_{0}^{2 \pi}\left(\chi_{\varepsilon} \partial_{\alpha}^{s} \theta^{\varepsilon}\right)\left(\chi_{\varepsilon} \theta_{\alpha}^{\varepsilon}\right)\left(\left[H, \chi_{\varepsilon} \theta_{\alpha}^{\varepsilon}\right]\left(\chi_{\varepsilon} \partial_{\alpha}^{s-1} \gamma^{\varepsilon}\right)\right) d \alpha \\
-\frac{8 \pi S c_{2}}{\left(L^{\varepsilon}\right)^{2}} \int_{0}^{2 \pi}\left(\chi_{\varepsilon} \partial_{\alpha}^{s} \theta^{\varepsilon}\right)\left(\chi_{\varepsilon} \theta_{\alpha}^{\varepsilon}\right)\left(\partial_{\alpha}^{s}\left(\mathbf{m}^{\varepsilon} \cdot \hat{\mathbf{t}}^{\varepsilon}\right)\right) d \alpha .
\end{gathered}
$$

We define $E_{3}$ and $E_{4}$ as

$$
\begin{gathered}
E_{3}=\frac{c_{3}(t)}{2} \int_{0}^{2 \pi}\left(\chi_{\varepsilon} \partial_{\alpha}^{s-1} \theta^{\varepsilon}\right)^{2} d \alpha \\
E_{4}=\frac{1}{2} \int_{0}^{2 \pi} \sqrt{c_{4}(\alpha, t)}\left(\partial_{\alpha}^{s-3} \gamma^{\varepsilon}\right) \Lambda\left(\sqrt{c_{4}(\alpha, t)}\left(\partial_{\alpha}^{s-3} \gamma^{\varepsilon}\right)\right) d \alpha .
\end{gathered}
$$

Note that $E_{4}$ is in the form $\int g \Lambda g d \alpha$, so we may make use of (1) when taking its time derivative. We will specify $c_{3}(t)$ and $c_{4}(\alpha, t)$ in short order; these will satisfy $c_{3} \geq 0$ and $c_{4} \geq 1$, so that $E_{3} \geq 0$, $E_{4} \geq 0$, and also so that derivatives of $\sqrt{c_{4}}$ remain bounded. These conditions ensure that (72) is satisfied. In order to take the time derivatives of $E_{3}$ and $E_{4}$, it is again helpful to have formulas for spatial derivatives of $\theta_{t}^{\varepsilon}$ and $\gamma_{t}^{\varepsilon}$ :

$$
\begin{gathered}
\partial_{\alpha}^{s-1} \theta_{t}^{\varepsilon}=\frac{2 \pi^{2}}{\left(L^{\varepsilon}\right)^{2}} \chi_{\varepsilon} H \partial_{\alpha}^{s} \gamma^{\varepsilon}+\Phi_{4} \\
\partial_{\alpha}^{s-3} \gamma_{t}^{\varepsilon}=-\widetilde{S}^{\varepsilon} \chi_{\varepsilon} \partial_{\alpha}^{s+1} \theta^{\varepsilon}+\Phi_{5}
\end{gathered}
$$

where $\Phi_{4}$ and $\Phi_{5}$ are given by

$$
\begin{gathered}
\Phi_{4}=\frac{2 \pi}{L^{\varepsilon}} \chi_{\varepsilon} \partial_{\alpha}^{s-1}\left(V_{W}^{\varepsilon}\left(\chi_{\varepsilon} \theta_{\alpha}^{\varepsilon}\right)\right)+\frac{2 \pi}{L^{\varepsilon}} \partial_{\alpha}^{s-1}\left(\mathbf{m}^{\varepsilon} \cdot \hat{\mathbf{n}}^{\varepsilon}\right), \\
\Phi_{5}=-\widetilde{S}^{\varepsilon} \chi_{\varepsilon} \partial_{\alpha}^{s-3}\left(Q_{1}^{\varepsilon}\left(\chi_{\varepsilon} \theta_{\alpha \alpha}^{\varepsilon}\right)\right)+\chi_{\varepsilon} \partial_{\alpha}^{s-3}\left(Q_{2}^{\varepsilon}\left(\chi_{\varepsilon} \gamma_{\alpha}^{\varepsilon}\right)\right)+\partial_{\alpha}^{s-3} Q_{3}^{\varepsilon} .
\end{gathered}
$$

We compute the time derivative of $E_{3}$ :

$$
\frac{d E_{3}}{d t}=\frac{d c_{3}}{d t} \cdot \frac{1}{2} \int_{0}^{2 \pi}\left(\chi_{\varepsilon} \partial_{\alpha}^{s-1} \theta^{\varepsilon}\right)^{2} d \alpha+\int_{0}^{2 \pi} c_{3}\left(\chi_{\varepsilon} \partial_{\alpha}^{s-1} \theta^{\varepsilon}\right)\left(\chi_{\varepsilon} \partial_{\alpha}^{s-1} \theta_{t}^{\varepsilon}\right) d \alpha .
$$

We substitute (91) into (95):

$$
\frac{d E_{3}}{d t}=\frac{2 \pi^{2}}{\left(L^{\varepsilon}\right)^{2}} \int_{0}^{2 \pi} c_{3}\left(\chi_{\varepsilon} \partial_{\alpha}^{s-1} \theta^{\varepsilon}\right)\left(\chi_{\varepsilon} H \partial_{\alpha}^{s} \gamma^{\varepsilon}\right) d \alpha+\Psi_{4},
$$


where $\Psi_{4}$ is defined as

$$
\Psi_{4}=\int_{0}^{2 \pi} c_{3}\left(\chi_{\varepsilon} \partial_{\alpha}^{s-1} \theta^{\varepsilon}\right)\left(\chi_{\varepsilon} \Phi_{4}\right) d \alpha+\frac{d c_{3}}{d t} \cdot \frac{1}{2} \int_{0}^{2 \pi}\left(\chi_{\varepsilon} \partial_{\alpha}^{s-1} \theta^{\varepsilon}\right)^{2} d \alpha .
$$

Next, we compute the time derivative of $E_{4}$ :

$$
\frac{d E_{4}}{d t}=\int_{0}^{2 \pi} \frac{\partial c_{4}}{\partial t} \cdot \frac{1}{2 \sqrt{c_{4}}}\left(\partial_{\alpha}^{s-3} \gamma^{\varepsilon}\right) \Lambda\left(\sqrt{c_{4}}\left(\partial_{\alpha}^{s-3} \gamma^{\varepsilon}\right)\right) d \alpha+\int_{0}^{2 \pi} \sqrt{c_{4}}\left(\partial_{\alpha}^{s-3} \gamma_{t}^{\varepsilon}\right) \Lambda\left(\sqrt{c_{4}}\left(\partial_{\alpha}^{s-3} \gamma^{\varepsilon}\right)\right) d \alpha .
$$

We comment now on the factor $\Lambda\left(\sqrt{c_{4}}\left(\partial_{\alpha}^{s-3} \gamma^{\varepsilon}\right)\right)$. We rewrite this first by using the definition $\Lambda=$ $H \partial_{\alpha}$, and the product rule:

$$
\Lambda\left(\sqrt{c_{4}}\left(\partial_{\alpha}^{s-3} \gamma^{\varepsilon}\right)\right)=H\left(\sqrt{c_{4}}\left(\partial_{\alpha}^{s-2} \gamma^{\varepsilon}\right)\right)+H\left(\frac{1}{2 \sqrt{c_{4}}}\left(\partial_{\alpha} c_{4}\right)\left(\partial_{\alpha}^{s-3} \gamma^{\varepsilon}\right)\right)
$$

Next, for the first term on the right-hand side, we pull $\sqrt{c_{4}}$ through the Hilbert transform, incurring a commutator:

$$
\Lambda\left(\sqrt{c_{4}}\left(\partial_{\alpha}^{s-3} \gamma^{\varepsilon}\right)\right)=\sqrt{c_{4}} H \partial_{\alpha}^{s-2} \gamma^{\varepsilon}+\left[H, \sqrt{c_{4}}\right]\left(\partial_{\alpha}^{s-2} \gamma^{\varepsilon}\right)+H\left(\frac{1}{\sqrt{c_{4}}}\left(\partial_{\alpha} c_{4}\right)\left(\partial_{\alpha}^{s-3} \gamma^{\varepsilon}\right)\right) .
$$

Now, making use of this formula, and substituting from (92), we find the following:

$$
\frac{d E_{4}}{d t}=-\frac{8 c_{4} \pi^{3} S}{\left(L^{\varepsilon}\right)^{3}} \int_{0}^{2 \pi}\left(\chi_{\varepsilon} \partial_{\alpha}^{s+1} \theta^{\varepsilon}\right)\left(\chi^{\varepsilon} H \partial_{\alpha}^{s-2} \gamma^{\varepsilon}\right) d \alpha+\Psi_{5},
$$

where $\Psi_{5}$ is defined as

$$
\begin{aligned}
\Psi_{5}= & \int_{0}^{2 \pi}\left(\sqrt{c_{4}} \widetilde{S}^{\varepsilon} \chi_{\varepsilon} \partial_{\alpha}^{s+1} \theta^{\varepsilon}\right) H\left(\frac{1}{2 \sqrt{c_{4}}}\left(\partial_{\alpha} c_{4}\right)\left(\partial_{\alpha}^{s-3} \gamma^{\varepsilon}\right)\right) d \alpha \\
+ & \int_{0}^{2 \pi}\left(\sqrt{c_{4}} \widetilde{S}^{\varepsilon} \chi_{\varepsilon} \partial_{\alpha}^{s+1} \theta^{\varepsilon}\right)\left[H, \sqrt{c_{4}}\right]\left(\partial_{\alpha}^{s-2} \gamma^{\varepsilon}\right) d \alpha+\int_{0}^{2 \pi}\left(\sqrt{c_{4}} \Phi_{5}\right) \Lambda\left(\sqrt{c_{4}}\left(\partial_{\alpha}^{s-3} \gamma^{\varepsilon}\right)\right) d \alpha \\
& +\int_{0}^{2 \pi} \frac{\partial c_{4}}{\partial t} \cdot \frac{1}{2 \sqrt{c_{4}}}\left(\partial_{\alpha}^{s-3} \gamma^{\varepsilon}\right) \Lambda\left(\sqrt{c_{4}}\left(\partial_{\alpha}^{s-3} \gamma^{\varepsilon}\right)\right) d \alpha .
\end{aligned}
$$

Next, we combine the time derivatives of $E_{1}, E_{2}, E_{3}$, and $E_{4}$. We integrate by parts in the integrals on the right-hand sides of (96) and (98), adding the results to (89):

$$
\begin{aligned}
\frac{d E_{1}}{d t}+\frac{d E_{2}}{d t}+\frac{d E_{3}}{d t}+\frac{d E_{4}}{d t} & \\
=\int_{0}^{2 \pi}\left[\frac{2 \pi S c_{2} \bar{c}_{1}}{L^{\varepsilon}}-\right. & \left.\frac{4 \pi^{3} S c_{2}}{\left(L^{\varepsilon}\right)^{3}}\left(\chi_{\varepsilon} \theta_{\alpha}^{\varepsilon}\right)^{2}-\frac{2 \pi^{2} c_{3}}{\left(L^{\varepsilon}\right)^{2}}+\frac{8 \pi^{3} S c_{4}}{\left(L^{\varepsilon}\right)^{3}}\right]\left(\chi_{\varepsilon} \partial_{\alpha}^{s} \theta^{\varepsilon}\right)\left(\chi_{\varepsilon} H \partial_{\alpha}^{s-1} \gamma^{\varepsilon}\right) \\
& +\frac{8 \pi^{3}}{\left(L^{\varepsilon}\right)^{3}} \int_{0}^{2 \pi}\left(\partial_{\alpha} c_{4}\right)\left(\chi_{\varepsilon} \partial_{\alpha}^{s} \theta^{\varepsilon}\right)\left(\chi_{\varepsilon} H \partial_{\alpha}^{s-2} \gamma^{\varepsilon}\right) d \alpha+\Psi_{3}+\Psi_{4}+\Psi_{5} .
\end{aligned}
$$

Then, we choose $c_{3}$ and $c_{4}$ so that the first integral on the right-hand side of (100) vanishes; recall that we have said $c_{4}=c_{4}(\alpha, t)$; this is because our choice of $c_{4}$ will involve $\left(\chi_{\varepsilon} \theta_{\alpha}^{\varepsilon}\right)^{2}$. Recall further 
that we have stated that we will choose $c_{4}$ so that $c_{4} \geq 1$; by bounding $c_{4}$ away from zero, we ensure that the factors of $\sqrt{c_{4}}$ which appear in denominators are not troublesome. Also recall that the constant $\bar{c}_{1}$ may be either positive or negative, while $c_{2}$ is positive. We write $\bar{c}_{1}$ using its positive and negative parts:

$$
\bar{c}_{1}=\left(\bar{c}_{1}\right)^{+}-\left(\bar{c}_{1}\right)^{-},
$$

with these defined the usual way $\left(\left(\bar{c}_{1}\right)^{+}=\max \left\{0, \bar{c}_{1}\right\}\right.$ and $\left(\bar{c}_{1}\right)^{-}=-\min \left\{0, \bar{c}_{1}\right\}$, so that $\left(\bar{c}_{1}\right)^{+} \geq 0$ and $\left.\left(\bar{c}_{1}\right)^{-} \geq 0\right)$. Then, we choose $c_{3}$ and $c_{4}$ so that $c_{3} \geq 0, c_{4} \geq 1$, and

$$
\frac{2 \pi S c_{2}\left(\bar{c}_{1}\right)^{+}}{L^{\varepsilon}}-\frac{2 \pi S c_{2}\left(\bar{c}_{1}\right)^{-}}{L^{\varepsilon}}-\frac{4 \pi^{3} S c_{2}}{\left(L^{\varepsilon}\right)^{3}}\left(\chi_{\varepsilon} \theta_{\alpha}^{\varepsilon}\right)^{2}-\frac{2 \pi^{2} c_{3}}{\left(L^{\varepsilon}\right)^{2}}+\frac{8 \pi^{3} S c_{4}}{\left(L^{\varepsilon}\right)^{3}}=0 .
$$

We can accomplish our desired goals with the following choices of $c_{3}$ and $c_{4}$ :

$$
\begin{gathered}
c_{3}=\frac{L^{\varepsilon} S c_{2}\left(\bar{c}_{1}\right)^{+}}{\pi}+\frac{4 \pi S}{L^{\varepsilon}}, \\
c_{4}=\frac{\left(L^{\varepsilon}\right)^{2} c_{2}\left(\bar{c}_{1}\right)^{-}}{4 \pi^{2}}+\frac{c_{2}}{2}\left(\chi_{\varepsilon} \theta_{\alpha}^{\varepsilon}\right)^{2}+1 .
\end{gathered}
$$

The remaining terms on the right-hand side of (100) can then be bounded in terms of the energy.

\subsection{Existence and regularity}

We now state and complete the proof of our existence theorem.

Theorem 11 Let $\left(\theta_{0}, \gamma_{0}\right) \in \mathcal{O}$ be given, with $\theta_{0}$ satisfying $\left\langle\sin \left(\theta_{0}\right)\right\rangle=0$. There exists $T>0$ and there exists $(\theta, \gamma) \in C([0, T] ; \overline{\mathcal{O}})$ such that $(\theta, \gamma)$ satisfies (25), (20) with $(\theta(\cdot, 0), \gamma(\cdot, 0))=\left(\theta_{0}, \gamma_{0}\right)$.

Note that the set $\overline{\mathcal{O}}$ denotes the closure of the open set $\mathcal{O}$.

Proof: Theorem 10 implies that the norm of solutions of the mollified problem, $\left(\theta^{\varepsilon}, \gamma^{\varepsilon}\right)$, cannot immediately blow up; indeed, the estimate (71) indicates that the solutions $\left(\theta^{\varepsilon}, \gamma^{\varepsilon}\right)$ are bounded independently of $\varepsilon$. By the continuation theorem for autonomous ODEs on a Banach space [31], this implies that the solutions all exist on a common time interval. We conclude there exists $T>0$ such that for all $\varepsilon>0$, solutions of the initial value problem $\left(\theta^{\varepsilon}, \gamma^{\varepsilon}\right)$ are in $C([0, T] \mathcal{O})$. (We note that to draw this conclusion, we must also check that the time derivative of the length is bounded and that the chord-arc condition continues to be satisfied. We note that these conclusions also follow from (71), since the time derivatives of these quantities can be controlled by the norm of $(\theta, \gamma)$.)

We have proved that there exists $T>0$ and $\left(\theta^{\varepsilon}, \gamma^{\varepsilon}\right) \in C([0, T] ; \mathcal{O})$ which solve the mollified evolution equations (41), with this $T$ independent of $\varepsilon$. Since $\mathcal{O}$ is a bounded subset of $H^{s} \times H^{s-3 / 2}$, and since we have taken $s$ to be sufficiently large, this implies that each of $\theta_{\alpha}^{\varepsilon}, \theta_{t}^{\varepsilon}, \gamma_{\alpha}^{\varepsilon}$, and $\gamma_{t}^{\varepsilon}$ are uniformly bounded periodic functions. Thus, $\theta^{\varepsilon}$ and $\gamma^{\varepsilon}$ are bounded, equicontinuous families. By the Arzela-Ascoli theorem, there exists $(\theta, \gamma) \in C([0,2 \pi] \times[0, T]) \times C([0,2 \pi] \times[0, T])$ such that a subsequence of $\left(\theta^{\varepsilon}, \gamma^{\varepsilon}\right)$ converges uniformly to $(\theta, \gamma)$ on $[0,2 \pi] \times[0, T]$. We will now show that this pair, $(\theta, \gamma)$, is in the closure of $\mathcal{O}$, and also that it solves the non-mollified evolution equation, (32).

Since each of $\theta$ and $\gamma$ are in $C([0,2 \pi] \times[0, T])$, we see that they are also in $L^{2}([0,2 \pi])$ at each time. By Lemma 1, we can conclude that the subsequence of $\left(\theta^{\varepsilon}, \gamma^{\varepsilon}\right)$ actually converges to $(\theta, \gamma)$ in $H^{s^{\prime}} \times H^{s^{\prime}-3 / 2}$, for any $s^{\prime}$ satisfying $3 / 2 \leq s^{\prime}<s$. For any such $s^{\prime}$, this implies $(\theta, \gamma) \in$ $L^{\infty}\left([0, T] ; H^{s^{\prime}} \times H^{s^{\prime}-3 / 2}\right)$. 
Since the solutions $\theta^{\varepsilon}$ all satisfy (70), we can pass to the limit, finding

$$
\left|q_{1}[\theta]\left(\alpha, \alpha^{\prime}\right)\right| \geq \bar{d}_{3}>0, \quad \forall \alpha, \alpha^{\prime} .
$$

Furthermore, the solutions $\theta^{\varepsilon}$ all satisfy

$$
\int_{0}^{2 \pi} \sin \left(\theta^{\varepsilon}\right) d \alpha=0
$$

since, along our subsequence, $\theta^{\varepsilon}$ converges uniformly to $\theta$, we can pass to the limit, finding $\mathbb{P}(\sin (\theta))=$ $\sin (\theta)$. We can pass to the limit as $\varepsilon$ vanishes (along our subsequence) in $\mu^{\varepsilon}$ as well. Recalling (42), and again in light of the regularity which we have already established, we see that

$$
\lim _{\varepsilon \rightarrow 0^{+}} \mu^{\varepsilon}=-\frac{L}{4 \pi^{2}} \int_{0}^{2 \pi} \mathcal{B} \cos (\theta) d \alpha=-\frac{1}{2 \pi} \int_{0}^{2 \pi}\left(U_{\alpha}+V \theta_{\alpha}\right) \cos (\theta) d \alpha .
$$

We have previously calculated this integral; it is equal to

$$
\frac{L_{t}}{2 \pi L} \int_{0}^{2 \pi} \sin (\theta) d \alpha
$$

Since we have seen that this integral is equal to zero, we see that $\mu^{\varepsilon}$ vanishes as $\varepsilon$ vanishes.

We now integrate (41) by integrating in time:

$$
\left(\theta^{\varepsilon}, \gamma^{\varepsilon}\right)=\left(\theta_{0}, \gamma_{0}\right)+\int_{0}^{t}\left(\mathcal{B}_{1}^{\varepsilon}+\mu^{\varepsilon}, \mathcal{B}_{2}^{\varepsilon}\right) d s
$$

for any $t \in[0, T]$. We have established sufficient regularity thus far to be able to pass to the limit (along our subsequence) here, finding

$$
(\theta, \gamma)=\left(\theta_{0}, \gamma_{0}\right)+\int_{0}^{t}\left(\mathcal{B}_{1}, \mathcal{B}_{2}\right) d s
$$

Differentiating this with respect to time, we have shown that $(\theta, \gamma)$ is indeed a solution of $(32)$, as desired.

It remains to demonstrate that $(\theta, \gamma) \in C\left([0, T] ; H^{s} \times H^{s-3 / 2}\right)$. For any $t \in[0, T]$, the sequence $\left(\theta^{\varepsilon}(\cdot, t), \gamma^{\varepsilon}(\cdot, t)\right)$ is uniformly bounded (with respect to both $\varepsilon$ and $\left.t\right)$ in $H^{s} \times H^{s-3 / 2}$. Since the unit ball of a Hilbert space is weakly compact, there exists a weak limit along a (further) subsequence. However, this weak limit must clearly be $(\theta, \gamma)$; this implies that $(\theta, \gamma) \in L^{\infty}\left([0, T] ; H^{s} \times H^{s-3 / 2}\right)$.

To show continuity in time in $H^{s} \times H^{s-3 / 2}$, we must show that for any $t_{*} \in[0, T]$,

$$
\lim _{t \rightarrow t_{*}}\left\|\theta(\cdot, t)-\theta\left(\cdot, t_{*}\right)\right\|_{s}+\left\|\gamma(\cdot, t)-\gamma\left(\cdot, t_{*}\right)\right\|_{s-3 / 2}=0 .
$$

(Of course, if $t_{*}=0$ or $t_{*}=T$, then the limit in (101) is to be taken as a one-sided limit.) We see in (101) that we are trying to establish convergence in a Hilbert space (i.e., we are showing that $(\theta(\cdot, t), \gamma(\cdot, t))$ converges to $\left(\theta\left(\cdot, t_{*}\right), \gamma\left(\cdot, t_{*}\right)\right)$ in $H^{s} \times H^{s-3 / 2}$, which is a Hilbert space). To establish convergence in a Hilbert space, it is sufficient to establish weak convergence, plus convergence of the norm.

For weak convergence, we focus on $\theta$, but there is no esential difference with $\gamma$. Let any $s^{\prime}$ satisfying $0<s^{\prime}<s$ be given. We know that $\theta(\cdot, t) \rightarrow \theta\left(\cdot, t_{*}\right)$ in $H^{s^{\prime}}$. Let $\phi \in H^{-s}$ be given. Since 
$0<s^{\prime}<s$, we have $-s<-s^{\prime}$, and thus $H^{-s^{\prime}}$ is dense in $H^{-s}$. Since we know that for all $t \in[0, T]$, we have $\theta(\cdot, t) \in H^{s}$, subject to the uniform bound that comes from the energy estimate, we can let $K$ denote the upper bound; so for any $t \in[0, T]$, we have $\|\theta(\cdot, t)\|_{H^{s}} \leq K$. Let $\delta>0$ be given. Choose $\phi_{\delta} \in H^{-s^{\prime}}$ be given such that $\left\|\phi-\phi_{\delta}\right\|_{H^{-s}} \leq \frac{\delta}{3(1+K)}$. Then we compute the following:

$$
\left\langle\theta(\cdot, t)-\theta\left(\cdot, t_{*}\right), \phi\right\rangle=\left\langle\theta(\cdot, t)-\theta\left(\cdot, t_{*}\right), \phi_{\delta}\right\rangle+\left\langle\theta(\cdot, t)-\theta\left(\cdot, t_{*}\right), \phi-\phi_{\delta}\right\rangle .
$$

The second term on the right-hand side is bounded by $\frac{2 K \delta}{3(1+K)} \leq \frac{2 \delta}{3}$. The first integral can be made smaller than $\frac{\delta}{3}$ by taking $t$ sufficiently close to $t_{*}$. This proves the weak convergence.

Finally, all that remains is to prove that the $H^{s} \times H^{s-3 / 2}$ norm of $(\theta, \gamma)$ is continuous in time. We omit the details of this, as the argument is identical to the corresponding argument in [3].

\section{Uniqueness and continuous dependence}

That solutions are unique follows from continuous dependence, so we will actually prove continuous dependence here. We have proved above that solutions $(\theta, \gamma)$ exist in the space $H^{s} \times H^{s-3 / 2}$. We therefore will assume that we have two solutions, $(\theta, \gamma)$ and $\left(\theta^{\prime}, \gamma^{\prime}\right)$ in this space, and estimate the difference in a lower-regularity space, which we choose as $H^{3} \times H^{3 / 2}$. This space is chosen to be high enough so that the estimates have positive powers of derivatives, but low enough so that the terms can be bounded by $\|\theta\|_{H^{s}}$ and $\|\gamma\|_{H^{s-3 / 2}}$.

Before beginning, we note that we will use the estimates in Lemma 7 with unregularized quantities, i.e., for $\varepsilon=0$. We also remark that the estimate that we now perform, for $\left(\theta-\theta^{\prime}, \gamma-\gamma^{\prime}\right)$ in $H^{3} \times H^{3 / 2}$, will be very similar to the energy estimate above for the existence proof.

Theorem 12 Let $\left(\theta_{0}, \gamma_{0}\right) \in \mathcal{O}$ and $\left(\theta_{0}^{\prime}, \gamma_{0}^{\prime}\right) \in \mathcal{O}$ be given, with $\left\langle\left\langle\sin \left(\theta_{0}\right)\right\rangle=\left\langle\left\langle\sin \left(\theta_{0}^{\prime}\right)\right\rangle=0\right.\right.$. If there exists $T>0$ such that there exists $(\theta, \gamma) \in C([0, T] ; \mathcal{O})$ which solves $(25)$, (31) with $(\theta(\cdot, 0), \gamma(\cdot, 0))=$ $\left(\theta_{0}, \gamma_{0}\right)$, and $\left(\theta^{\prime}, \gamma^{\prime}\right) \in C([0, T] ; \mathcal{O})$ which solves $(25)$, (31) with $\left(\theta^{\prime}(\cdot, 0), \gamma^{\prime}(\cdot, 0)\right)=\left(\theta_{0}^{\prime}, \gamma_{0}^{\prime}\right)$, then there exists $c>0$ such that

$$
\sup _{t \in[0, T]}\left(\left|L-L^{\prime}\right|+\left\|\theta-\theta^{\prime}\right\|_{H^{3}}+\left\|\gamma-\gamma^{\prime}\right\|_{H^{3 / 2}}\right) \leq c\left(\left|L(0)-L^{\prime}(0)\right|+\left\|\theta_{0}-\theta_{0}^{\prime}\right\|_{H^{3}}+\left\|\gamma_{0}-\gamma_{0}^{\prime}\right\|_{H^{3 / 2}}\right) .
$$

Moreover, the solution of the initial value problem (25), (31) with initial data $\left(\theta_{0}, \gamma_{0}\right) \in \mathcal{O}$ is unique.

Proof: We define $E_{d}$ to be

$$
E_{d}=Z_{0}+Z_{1}+Z_{2}+Z_{3}+Z_{4}
$$

where

$$
\begin{gathered}
Z_{0}=\frac{1}{2}\left(L-L^{\prime}\right)^{2}+\frac{1}{2} \int_{0}^{2 \pi}\left(\theta-\theta^{\prime}\right)^{2}+\left(\gamma-\gamma^{\prime}\right)^{2} d \alpha \\
Z_{1}=\frac{d_{1}(t)}{2} \int_{0}^{2 \pi}\left(\partial_{\alpha}^{3} \theta-\partial_{\alpha}^{3} \theta^{\prime}\right)^{2} d \alpha \\
Z_{2}=\frac{d_{2}(t)}{2} \int_{0}^{2 \pi}\left(\partial_{\alpha} \gamma-\partial_{\alpha} \gamma^{\prime}\right) H\left(\partial_{\alpha}^{2} \gamma-\partial_{\alpha}^{2} \gamma^{\prime}\right) d \alpha
\end{gathered}
$$


The coefficients $d_{1}$ and $d_{2}$ will be chosen as we make the estimate. We will also define $Z_{3}$ and $Z_{4}$ in due course. We note, however, that (as in the proof of Theorem 10), all of these choices will be made to ensure that $E_{d}$ controls a norm; in particular, we will have

$$
\frac{1}{2}\left(\left\|\theta-\theta^{\prime}\right\|_{H^{3}}^{2}+\left\|\gamma-\gamma^{\prime}\right\|_{H^{3 / 2}}^{2}\right) \leq E_{d} .
$$

Before we begin to estimate $E_{d}$, we discuss the strategy. We will show that if $(\theta, \gamma)$ and $\left(\theta^{\prime}, \gamma^{\prime}\right)$ are in $\mathcal{O}$, then

$$
\frac{d E_{d}}{d t} \leq c E_{d}
$$

Solving this inequality, we find

$$
E_{d}(t) \leq E_{d}(0) e^{c t} .
$$

This implies both continuous dependence and uniqueness. If we want $E_{d}(t)$ to be small, then we can achieve this by taking $E_{d}(0)$ to be sufficiently small. This is for the $H^{3} \times H^{3 / 2}$ norm; higher norms follow by interpolation. This is the continuous dependence. For uniqueness, we note that if $E_{d}(0)=0$, then $E_{d}(t)=0$, so we have $\theta=\theta^{\prime}$ and $\gamma=\gamma^{\prime}$.

It is important to be able to estimate differences of the various quantities associated to $(\theta, \gamma)$ and $\left(\theta^{\prime}, \gamma^{\prime}\right)$, so we will make such estimates now, before beginning the estimate of $E_{d}$. The simplest associated quantites are the unit tangent and normal vectors; the following bounds follow from standard Lipschitz estimates for sine and cosine:

$$
\left\|\hat{\mathbf{t}}-\hat{\mathbf{t}}^{\prime}\right\|_{H^{3}}=\left\|\left(\cos (\theta)-\cos \left(\theta^{\prime}\right), \sin (\theta)-\sin \left(\theta^{\prime}\right)\right)\right\|_{H^{3}} \leq c\left\|\theta-\theta^{\prime}\right\|_{H^{3}} \leq c E_{d}^{1 / 2},
$$

and similarly,

$$
\left\|\hat{\mathbf{n}}-\hat{\mathbf{n}}^{\prime}\right\|_{H^{3}} \leq c E_{d}^{1 / 2} .
$$

Then, since $z_{\alpha}=\frac{L}{2 \pi} \hat{\mathbf{t}}$, a bound for $z_{\alpha}-z_{\alpha}^{\prime}$ follows:

$$
\left\|\Phi^{-1}\left(z_{\alpha}-z_{\alpha}^{\prime}\right)\right\|_{H^{3}} \leq\left\|\left(\frac{L-L^{\prime}}{2 \pi}\right) \hat{\mathbf{t}}\right\|_{H^{3}}+\frac{L^{\prime}}{2 \pi}\left\|\hat{\mathbf{t}}-\hat{\mathbf{t}}^{\prime}\right\|_{H^{3}} \leq c E_{d}^{1 / 2} .
$$

Next, we estimate $\mathbf{m}-\mathbf{m}^{\prime}$. We write

$$
\Phi\left(\mathbf{m}-\mathbf{m}^{\prime}\right)^{*}=I+I I,
$$

with the definitions

$$
\begin{gathered}
I=z_{\alpha} \mathcal{K}\left[z_{d}\right]\left(\left(\frac{\gamma}{z_{\alpha}}\right)_{\alpha}\right)-z_{\alpha}^{\prime} \mathcal{K}\left[z^{\prime}\right]\left(\left(\frac{\gamma^{\prime}}{z_{\alpha}^{\prime}}\right)_{\alpha}\right) \\
I I=\frac{z_{\alpha}}{2 i}\left[H, \frac{1}{z_{\alpha}^{2}}\right]\left(z_{\alpha}\left(\frac{\gamma}{z_{\alpha}}\right)_{\alpha}\right)-\frac{z_{\alpha}^{\prime}}{2 i}\left[H, \frac{1}{\left(z_{\alpha}^{\prime}\right)^{2}}\right]\left(z_{\alpha}^{\prime}\left(\frac{\gamma^{\prime}}{z_{\alpha}^{\prime}}\right)_{\alpha}\right) .
\end{gathered}
$$

We rewrite $I$ by adding and subtracting:

$$
I=\left(z_{\alpha}-z_{\alpha}^{\prime}\right) \mathcal{K}\left[z_{d}\right]\left(\left(\frac{\gamma}{z_{\alpha}}\right)_{\alpha}\right)+z_{\alpha}^{\prime}\left(\mathcal{K}\left[z_{d}\right]-\mathcal{K}\left[z^{\prime}\right]\right)\left(\left(\frac{\gamma}{z_{\alpha}}\right)_{\alpha}\right)+z_{\alpha}^{\prime} \mathcal{K}\left[z^{\prime}\right]\left(\left(\frac{\gamma}{z_{\alpha}}\right)_{\alpha}-\left(\frac{\gamma^{\prime}}{z_{\alpha}^{\prime}}\right)_{\alpha}\right) .
$$


With the assumed uniform bounds on $\theta$ and $\gamma$, and using (103), the first term on the right-hand side is bounded by $c E_{d}^{1 / 2}$. For the second term, we apply Lemma 3 , and we thus see that this term is also bounded by $c E_{d}^{1 / 2}$. For the third term on the right-hand side, its norm in $H^{3}$ is bounded by $c E_{d}^{1 / 2}$ since $\mathcal{K}\left[z^{\prime}\right]$ is a smoothing operator (i.e., we use Lemma 2 ).

We now turn our attention to the term $I I$. We start by adding and subtracting:

$$
\begin{aligned}
I I=\frac{z_{\alpha}-z_{\alpha}^{\prime}}{2 i}\left[H, \frac{1}{z_{\alpha}^{2}}\right]\left(z_{\alpha}\left(\frac{\gamma}{z_{\alpha}}\right)_{\alpha}\right)+\frac{z_{\alpha}^{\prime}}{2 i}\left[H, \frac{1}{z_{\alpha}^{2}}-\frac{1}{\left(z_{\alpha}^{\prime}\right)^{2}}\right] & \left(z_{\alpha}\left(\frac{\gamma}{z_{\alpha}}\right)_{\alpha}\right) \\
& +\frac{z_{\alpha}^{\prime}}{2 i}\left[H, \frac{1}{\left(z_{\alpha}^{\prime}\right)^{2}}\right]\left(z_{\alpha}\left(\frac{\gamma}{z_{\alpha}}\right)_{\alpha}-z_{\alpha}^{\prime}\left(\frac{\gamma^{\prime}}{z_{\alpha}^{\prime}}\right)_{\alpha}\right) .
\end{aligned}
$$

The first term on the right-hand side can be immediately bounded by $c E_{d}^{1 / 2}$. The third term can be bounded by $c E_{d}^{1 / 2}$ by using Lemma 4 . For the second term on the right-hand side, we bound it not by using smoothing properties of commutators, but by not regarding it as a commutator at all. That is, we write the term out, letting the function $f$ temporarily denote $f=z_{\alpha}\left(\gamma / z_{\alpha}\right)_{\alpha}$ :

$$
\left[H, \frac{1}{z_{\alpha}^{2}}-\frac{1}{\left(z_{\alpha}^{\prime}\right)^{2}}\right] f=H\left(\left(\frac{1}{z_{\alpha}^{2}}-\frac{1}{\left(z_{\alpha}^{\prime}\right)^{2}}\right) f\right)-\left(\frac{1}{z_{\alpha}^{2}}-\frac{1}{\left(z_{\alpha}^{\prime}\right)^{2}}\right) H f .
$$

Each of the terms on the right-hand side can clearly be bounded by $c E_{d}^{1 / 2}$. This completes the estimate of $\mathbf{m}-\mathbf{m}$; we conclude

$$
\left\|\mathbf{m}-\mathbf{m}^{\prime}\right\|_{H^{3}} \leq c E_{d}^{1 / 2} .
$$

We next estimate $V_{W}-V_{W}^{\prime}$. From formula (24), after adding and subtracting several times, and using the above estimates for $\hat{\mathbf{t}}-\hat{\mathbf{t}}^{\prime}$ and $\mathbf{m}-\mathbf{m}^{\prime}$, the following estimate can be found:

$$
\left\|V_{W}-V_{W}^{\prime}\right\|_{H^{2}} \leq c E_{d}^{1 / 2} \text {. }
$$

We omit the details of most other differences to be estimated, since they are similar to the above. However, we do remark that one interesting estimate is for the difference $\left(I+2 A \mathcal{J}\left[z_{d}\right]\right)^{-1}-(I+$ $\left.2 A \mathcal{J}\left[z_{d}^{\prime}\right]\right)^{-1}$. We consider invertible linear operators $B_{1}$ and $B_{2}$, and we see that

$$
B_{1}^{-1}-B_{2}^{-1}=B_{1}^{-1} B_{2} B_{2}^{-1}-B_{1}^{-1} B_{1} B_{2}^{-1}=B_{1}^{-1}\left(B_{2}-B_{1}\right) B_{2}^{-1} .
$$

Thus, we see that we can make a Lipschitz estimate for the inverses by estimating the inverses individually and by making a Lipschitz estimate for the forward operators. If we let $B_{1}=I+2 A \mathcal{J}\left[z_{d}\right]$ and $B_{2}=I+2 A \mathcal{J}\left[z_{d}^{\prime}\right]$, then $B_{1}$ and $B_{2}$ are bounded invertible operators by Lemma 6 , and we see from (106) that we need to be able to estimate $B_{2}-B_{1}=2 A\left(\mathcal{J}\left[z_{d}^{\prime}\right]-\mathcal{J}\left[z_{d}\right]\right)$. Since we can decompose $\mathcal{J}$ as commutators plus terms involving $\mathcal{K}$, and since we know how to make Lipschitz estimates for both of these kinds of terms, we are able to make the estimate for the difference.

We can use these estimates to conclude

$$
\frac{d Z_{0}}{d t} \leq c E_{d}
$$

Now, we get to the most important part of the proof of the theorem, which is to take the time derivative of $Z_{1}$ and $Z_{2}$. We have

$$
\frac{d Z_{1}}{d t}=d_{1} \int_{0}^{2 \pi}\left(\partial_{\alpha}^{3} \theta-\partial_{\alpha}^{3} \theta^{\prime}\right)\left(\partial_{\alpha}^{3} \theta_{t}-\partial_{\alpha}^{3} \theta_{t}^{\prime}\right) d \alpha+\frac{d d_{1}}{d t} \cdot \frac{1}{2} \int_{0}^{2 \pi}\left(\partial_{\alpha}^{3} \theta-\partial_{\alpha}^{3} \theta^{\prime}\right)^{2} d \alpha .
$$


Applying $\partial_{\alpha}^{3}$ to (25), we get

$$
\partial_{\alpha}^{3} \theta_{t}=\frac{2 \pi^{2}}{L^{2}} H \partial_{\alpha}^{4} \gamma+\frac{2 \pi}{L}\left(\partial_{\alpha}^{3} V_{W}\right) \theta_{\alpha}+\frac{2 \pi}{L} V_{W}\left(\partial_{\alpha}^{4} \theta\right)+Y_{1},
$$

where $Y_{1}$ is defined as

$$
Y_{1}=\frac{6 \pi}{L}\left(\partial_{\alpha}^{2} V_{W}\right)\left(\partial_{\alpha}^{2} \theta\right)+\frac{6 \pi}{L}\left(\partial_{\alpha} V_{W}\right)\left(\partial_{\alpha}^{3} \theta\right)+\frac{2 \pi}{L} \partial_{\alpha}^{3}(\mathbf{m} \cdot \hat{\mathbf{n}}) .
$$

Of course, we get the corresponding formula for $\partial_{\alpha}^{3} \theta^{\prime}$. These considerations allow us to substitute into (108):

$$
\begin{gathered}
\frac{d Z_{1}}{d t}=d_{1} \int_{0}^{2 \pi}\left(\partial_{\alpha}^{3} \theta-\partial_{\alpha}^{3} \theta^{\prime}\right)\left(\frac{2 \pi^{2}}{L^{2}} H \partial_{\alpha}^{4} \gamma-\frac{2 \pi^{2}}{\left(L^{\prime}\right)^{2}} H \partial_{\alpha}^{4} \gamma^{\prime}\right) d \alpha \\
+d_{1} \int_{0}^{2 \pi}\left(\partial_{\alpha}^{3} \theta-\partial_{\alpha}^{3} \theta^{\prime}\right)\left(\frac{2 \pi}{L}\left(\partial_{\alpha}^{3} V_{W}\right) \theta_{\alpha}-\frac{2 \pi}{L^{\prime}}\left(\partial_{\alpha}^{3} V_{W}^{\prime}\right) \theta_{\alpha}^{\prime}\right) d \alpha+d_{1} \int_{0}^{2 \pi}\left(\partial_{\alpha}^{3} \theta-\partial_{\alpha}^{3} \theta^{\prime}\right)\left(\frac{2 \pi}{L} V_{W}\left(\partial_{\alpha}^{4} \theta\right)-\frac{2 \pi}{L^{\prime}} V_{W}^{\prime}\left(\partial_{\alpha}^{4} \theta^{\prime}\right)\right) d \alpha \\
+d_{1} \int_{0}^{2 \pi}\left(\partial_{\alpha}^{3} \theta-\partial_{\alpha}^{3} \theta^{\prime}\right)\left(Y_{1}-Y_{1}^{\prime}\right) d \alpha+\frac{d d_{1}}{d t} \cdot \frac{1}{2} \int_{0}^{2 \pi}\left(\partial_{\alpha}^{3} \theta-\partial_{\alpha}^{3} \theta^{\prime}\right)^{2} d \alpha . \quad(109)
\end{gathered}
$$

There are five integrals on the right-hand side of (109), and we give these names; we write

$$
\frac{d Z_{1}}{d t}=\Upsilon_{1}+\Upsilon_{2}+\Upsilon_{3}+\Upsilon_{4}+\Upsilon_{5}
$$

We must manipulate and estimate these, and we begin now with $\Upsilon_{1}$.

We add and subtract to write $\Upsilon_{1}$ as follows:

$\Upsilon_{1}=d_{1} \int_{0}^{2 \pi}\left(\partial_{\alpha}^{3} \theta-\partial_{\alpha}^{3} \theta^{\prime}\right)\left(\frac{2 \pi^{2}}{L^{2}}\right)\left(H \partial_{\alpha}^{4} \gamma-H \partial_{\alpha}^{4} \gamma^{\prime}\right) d \alpha+d_{1} \int_{0}^{2 \pi}\left(\partial_{\alpha}^{3} \theta-\partial_{\alpha}^{3} \theta^{\prime}\right)\left(\frac{2 \pi^{2}}{L^{2}}-\frac{2 \pi^{2}}{\left(L^{\prime}\right)^{2}}\right) H \partial_{\alpha}^{4} \gamma^{\prime} d \alpha$.

Of the two integrals on the right-hand side, the second can clearly be bounded in terms of $E_{d}$ since the definition of $Z_{0}$ includes $\left(L-L^{\prime}\right)^{2}$ (and recall that $L$ and $L^{\prime}$ must both be greater than or equal to $2 \pi)$. Therefore, we have

$$
\Upsilon_{1} \leq d_{1} \int_{0}^{2 \pi}\left(\partial_{\alpha}^{3} \theta-\partial_{\alpha}^{3} \theta^{\prime}\right)\left(\frac{2 \pi^{2}}{L^{2}}\right)\left(H \partial_{\alpha}^{4} \gamma-H \partial_{\alpha}^{4} \gamma^{\prime}\right) d \alpha+c E_{d}
$$

To work with $\Upsilon_{2}$, we first expand $\partial_{\alpha}^{3} V_{W}$ :

$$
\partial_{\alpha}^{3} V_{W}=\frac{\pi}{L} \theta_{\alpha} H \gamma_{\alpha \alpha}+\frac{\pi}{L}\left[H, \theta_{\alpha}\right]\left(\gamma_{\alpha \alpha}\right)+\frac{2 \pi}{L} H\left(\gamma_{\alpha} \theta_{\alpha \alpha}\right)+\frac{\pi}{L} H\left(\gamma \partial_{\alpha}^{3} \theta\right)-\partial_{\alpha}^{2}(\mathbf{m} \cdot \hat{\mathbf{t}}) .
$$

After some adding and subtracting, the leading-order term of

$$
\frac{2 \pi}{L}\left(\partial_{\alpha}^{3} V_{W}\right) \theta_{\alpha}-\frac{2 \pi}{L^{\prime}}\left(\partial_{\alpha}^{3} V_{W}^{\prime}\right) \theta_{\alpha}^{\prime}
$$

can then be seen to be

$$
\frac{2 \pi^{2}}{L^{2}} \theta_{\alpha}^{2}\left(H \gamma_{\alpha \alpha}-H \gamma_{\alpha \alpha}^{\prime}\right)
$$


indeed, the contributions to $\Upsilon_{2}$ from all the other terms can then be bounded in terms of $E_{d}$. These considerations yield the following:

$$
\Upsilon_{2} \leq d_{1} \int_{0}^{2 \pi}\left(\partial_{\alpha}^{3} \theta-\partial_{\alpha}^{3} \theta^{\prime}\right)\left(\frac{2 \pi^{2}}{L^{2}} \theta_{\alpha}^{2}\right)\left(H \partial_{\alpha}^{2} \gamma-H \partial_{\alpha}^{2} \gamma^{\prime}\right) d \alpha+c E_{d}
$$

Each of $\Upsilon_{3}, \Upsilon_{4}$, and $\Upsilon_{5}$ are bounded by $c E_{d}$. For $\Upsilon_{4}$, this follows from the estimates such as (104) and (105). For $\Upsilon_{5}$, the fact that it can be bounded by $c E_{d}$ follows immediately since $\frac{d d_{1}}{d t}$ is bounded; of course, we have not yet defined $d_{1}$, but once we do, it will be clear that $d_{1}$ and $\frac{d d_{1}}{d t}$ are bounded. To be slightly more precise, $d_{1}$, when we do define it, will be in terms of $(\theta, \gamma)$ only, and we have assumed that $(\theta, \gamma)$ is a bounded solution.

For $\Upsilon_{3}$, we add and subtract to reach the following:

$\Upsilon_{3}=d_{1} \int_{0}^{2 \pi}\left(\partial_{\alpha}^{3} \theta-\partial_{\alpha}^{3} \theta^{\prime}\right)\left(\frac{2 \pi}{L} V_{W}\right)\left(\partial_{\alpha}^{4} \theta-\partial_{\alpha}^{4} \theta^{\prime}\right) d \alpha+d_{1} \int_{0}^{2 \pi}\left(\partial_{\alpha}^{3} \theta-\partial_{\alpha}^{3} \theta^{\prime}\right)\left(\frac{2 \pi}{L} V_{W}-\frac{2 \pi}{L^{\prime}} V_{W}^{\prime}\right)\left(\partial_{\alpha}^{4} \theta^{\prime}\right) d \alpha$.

The first of these can be integrated by parts:

$$
\Upsilon_{3}=\frac{d_{1}}{2} \int_{0}^{2 \pi}\left(\frac{2 \pi}{L} \partial_{\alpha} V_{W}\right)\left(\partial_{\alpha}^{3} \theta-\partial_{\alpha} \theta^{\prime}\right)^{2} d \alpha .+d_{1} \int_{0}^{2 \pi}\left(\partial_{\alpha}^{3} \theta-\partial_{\alpha}^{3} \theta^{\prime}\right)\left(\frac{2 \pi}{L} V_{W}-\frac{2 \pi}{L^{\prime}} V_{W}^{\prime}\right)\left(\partial_{\alpha}^{4} \theta^{\prime}\right) d \alpha .
$$

Both of these terms, then, can be bounded by $c E_{d}$. So far, then, we have calculated

$$
\begin{aligned}
\frac{d Z_{1}}{d t} & =\Upsilon_{1}+\Upsilon_{2}+\Upsilon_{3}+\Upsilon_{4}+\Upsilon_{5} \leq c E_{d} \\
& +\int_{0}^{2 \pi}\left(\frac{2 \pi^{2} d_{1}}{L^{2}}\right)\left(\partial_{\alpha}^{3}\left(\theta-\theta^{\prime}\right)\right) H \partial_{\alpha}^{4}\left(\gamma-\gamma^{\prime}\right) d \alpha+\int_{0}^{2 \pi}\left(\frac{2 \pi^{2} d_{1} \theta_{\alpha}^{2}}{L^{2}}\right)\left(\partial_{\alpha}^{3}\left(\theta-\theta^{\prime}\right)\right) H \partial_{\alpha}^{2}\left(\gamma-\gamma^{\prime}\right) d \alpha .
\end{aligned}
$$

We now are ready to take the time derivative of $Z_{2}$. To begin, we have

$$
\frac{d Z_{2}}{d t}=d_{2} \int_{0}^{2 \pi}\left(\gamma_{\alpha t}-\gamma_{\alpha t}^{\prime}\right)\left(H \partial_{\alpha}^{2} \gamma-H \partial_{\alpha}^{2} \gamma^{\prime}\right) d \alpha+\frac{d d_{2}}{d t} \cdot \frac{1}{2} \int_{0}^{2 \pi}\left(\gamma_{\alpha}-\gamma_{\alpha}^{\prime}\right)\left(H \partial_{\alpha}^{2} \gamma-H \partial_{\alpha}^{2} \gamma^{\prime}\right) d \alpha .
$$

We take the derivative of $\gamma_{t}$, finding

$$
\partial_{\alpha} \gamma_{t}=-\left(\frac{8 \pi^{3} S}{L^{3}}\right) \partial_{\alpha}^{5} \theta-\left(\frac{8 \pi^{3} S}{L^{3}}\right)\left(\partial_{\alpha}^{3} \theta\right)\left(\frac{3}{2} \theta_{\alpha}^{2}-\frac{L^{2} \overline{c_{1}}}{4 \pi^{2}}\right)+\gamma_{\alpha \alpha} Q_{2}+Y_{2},
$$

where $Y_{2}$ denotes the following collection of terms:

$$
Y_{2}=-\widetilde{S} \theta_{\alpha \alpha} \partial_{\alpha} Q_{1}+\gamma_{\alpha} \partial_{\alpha} Q_{2}+\partial_{\alpha} Q_{3} .
$$

We dispense with many of the details; we only get two significant terms from $\frac{d Z_{2}}{d t}$, and these correspond to the first two terms on the right-hand side of (110). We get the following estimate:

$$
\begin{aligned}
& \frac{d Z_{2}}{d t} \leq c E_{d}+\int_{0}^{2 \pi}\left(-\frac{8 \pi^{3} S d_{2}}{L^{3}}\right)\left(\partial_{\alpha}^{5}\left(\theta-\theta^{\prime}\right)\right)\left(H \partial_{\alpha}^{2}\left(\gamma-\gamma^{\prime}\right)\right) d \alpha \\
&+\int_{0}^{2 \pi}\left(-\frac{12 \pi^{3} S d_{2} \theta_{\alpha}^{2}}{L^{3}}+\frac{2 \pi S d_{2} \bar{c}_{1}}{L}\right)\left(\partial_{\alpha}^{3}\left(\theta-\theta^{\prime}\right)\right)\left(H \partial_{\alpha}^{2}\left(\gamma-\gamma^{\prime}\right)\right) d \alpha
\end{aligned}
$$


We choose $d_{1}$ and $d_{2}$ so that

$$
\frac{2 \pi^{2} d_{1}}{L^{2}}-\frac{8 \pi^{3} S d_{2}}{L^{3}}=0
$$

Specifically, we make the choices

$$
d_{2}=\max \left\{1, \frac{1}{S}\right\}, \quad d_{1}=\frac{4 \pi S d_{2}}{L} .
$$

Then, when adding $\frac{d Z_{1}}{d t}$ and $\frac{d Z_{2}}{d t}$, the leading terms cancel, leaving the following:

$$
\frac{d Z_{1}}{d t}+\frac{d Z_{2}}{d t} \leq c E_{d}+\int_{0}^{2 \pi}\left(\frac{2 \pi^{2} d_{1} \theta_{\alpha}^{2}}{L^{2}}-\frac{12 \pi^{3} S d_{2} \theta_{\alpha}^{2}}{L^{3}}+\frac{2 \pi S d_{2} \bar{c}_{1}}{L}\right)\left(\partial_{\alpha}^{3}\left(\theta-\theta^{\prime}\right)\right)\left(H \partial_{\alpha}^{2}\left(\gamma-\gamma^{\prime}\right)\right) d \alpha
$$

Using the definition of $d_{1}$, this simplifies:

$$
\frac{d Z_{1}}{d t}+\frac{d Z_{2}}{d t} \leq c E_{d}+\int_{0}^{2 \pi}\left(-\frac{4 \pi^{3} S d_{2} \theta_{\alpha}^{2}}{L^{3}}+\frac{2 \pi S d_{2} \bar{c}_{1}}{L}\right)\left(\partial_{\alpha}^{3}\left(\theta-\theta^{\prime}\right)\right)\left(H \partial_{\alpha}^{2}\left(\gamma-\gamma^{\prime}\right)\right) d \alpha .
$$

We now define $Z_{3}$, and we will soon define $Z_{4}$; these terms will allow us to cancel the integral on the right-hand side of (113); notice that this integral is not bounded in terms of $E_{d}$, since it has too many derivatives. We let $Z_{3}$ be given by

$$
Z_{3}=\frac{d_{3}(t)}{2} \int_{0}^{2 \pi}\left(\partial_{\alpha}^{2} \theta-\partial_{\alpha}^{2} \theta^{\prime}\right)^{2} d \alpha
$$

Taking its time derivative, we get

$$
\frac{d Z_{3}}{d t}=d_{3} \int_{0}^{2 \pi}\left(\partial_{\alpha}^{2} \theta_{t}-\partial_{\alpha}^{2} \theta_{t}^{\prime}\right)\left(\partial_{\alpha}^{2} \theta-\partial_{\alpha}^{2} \theta^{\prime}\right) d \alpha+\frac{d d_{3}}{d t} \cdot \frac{1}{2} \int_{0}^{2 \pi}\left(\partial_{\alpha}^{2} \theta-\partial_{\alpha}^{2} \theta^{\prime}\right) d \alpha .
$$

Applying two spatial derivatives to $\theta_{t}$, we get the following:

$$
\partial_{\alpha}^{2} \theta_{t}=\frac{2 \pi^{2}}{L^{2}} H \partial_{\alpha}^{3} \gamma+Y_{3}
$$

where the term $Y_{3}$ is defined as

$$
Y_{3}=\partial_{\alpha}^{2}\left(\frac{2 \pi}{L} V_{W} \theta_{\alpha}+\frac{2 \pi}{L} \mathbf{m} \cdot \hat{\mathbf{n}}\right) .
$$

As above, we do not include all details, but there is only one term which is not bounded in terms of $E_{d}$ :

$$
\frac{d Z_{3}}{d t} \leq c E_{d}+\int_{0}^{2 \pi}\left(\frac{2 \pi^{2} d_{3}}{L^{2}}\right)\left(H \partial_{\alpha}^{3}\left(\gamma-\gamma^{\prime}\right)\right)\left(\partial_{\alpha}^{2}\left(\theta-\theta^{\prime}\right)\right) d \alpha .
$$

In the integral on the right-hand side, we integrate by parts once:

$$
\frac{d Z_{3}}{d t} \leq c E_{d}+\int_{0}^{2 \pi}\left(-\frac{2 \pi^{2} d_{3}}{L^{2}}\right)\left(H \partial_{\alpha}^{2}\left(\gamma-\gamma^{\prime}\right)\right)\left(\partial_{\alpha}^{3}\left(\theta-\theta^{\prime}\right)\right) d \alpha .
$$

For the final piece of $E_{d}$, we define $Z_{4}$. Before doing so, we note that the coefficients $d_{1}, d_{2}$, and $d_{3}$ that we have introduced thus far are functions of $t$ only. Now, however, the term $Z_{4}$ will involve 
a coefficient $d_{4}$ which we must take to depend on both $\alpha$ and $t$; this is because it will be used to cancel terms in (112) which involve $\theta_{\alpha}^{2}$. With this in mind, our definition is

$$
Z_{4}=\frac{1}{2} \int_{0}^{2 \pi}\left(\sqrt{d_{4}(\alpha, t)}\left(\gamma-\gamma^{\prime}\right)\right) H \partial_{\alpha}\left(\sqrt{d_{4}(\alpha, t)}\left(\gamma-\gamma^{\prime}\right)\right) d \alpha .
$$

We note that the form of $Z_{4}$ may at first look unusual; we note, however, that it is in the form $\int g \Lambda g d \alpha$, with $g=\sqrt{d_{4}}\left(\gamma-\gamma^{\prime}\right)$, and we may thus use (1) to estimate its time derivative. Furthermore, we will choose $d_{4}$ to satisfy $d_{4} \geq 1$ so that it is clear that derivatives of $\sqrt{d_{4}}$ are bounded. Taking the time derivative of $Z_{4}$, we get

$$
\frac{d Z_{4}}{d t}=\int_{0}^{2 \pi} \sqrt{d_{4}}\left(\gamma_{t}-\gamma_{t}^{\prime}\right) H \partial_{\alpha}\left(\sqrt{d_{4}}\left(\gamma-\gamma^{\prime}\right)\right) d \alpha+\int_{0}^{2 \pi} \frac{d d_{4}}{d t} \cdot \frac{1}{2 \sqrt{d_{4}}}\left(\gamma-\gamma^{\prime}\right) H \partial_{\alpha}\left(\sqrt{d_{4}}\left(\gamma-\gamma^{\prime}\right)\right) d \alpha .
$$

As for $Z_{3}$, when considering $Z_{4}$, there is only one significant term, and this is from the leading-order term in (31). After our usual adding and subtracting, we arrive at

$$
\frac{d Z_{4}}{d t} \leq c E_{d}+\int_{0}^{2 \pi}\left(-\frac{8 \pi^{3} S}{L^{3}}\right) \sqrt{d_{4}}\left(\partial_{\alpha}^{4}\left(\theta-\theta^{\prime}\right)\right) H \partial_{\alpha}\left(\sqrt{d_{4}}\left(\gamma-\gamma^{\prime}\right)\right) d \alpha .
$$

We are able to pass the second factor of $\sqrt{d_{4}}$ above through $H \partial_{\alpha}$, incurring only terms which can be bounded by $c E_{d}$. We are left, then with

$$
\frac{d Z_{4}}{d t} \leq c E_{d}+\int_{0}^{2 \pi}\left(-\frac{8 \pi^{3} S d_{4}}{L^{3}}\right)\left(\partial_{\alpha}^{4}\left(\theta-\theta^{\prime}\right)\right) H \partial_{\alpha}\left(\gamma-\gamma^{\prime}\right) d \alpha .
$$

We integrate by parts in this integral, retaining just one integral that is not bounded by $c E_{d}$ :

$$
\frac{d Z_{4}}{d t} \leq c E_{d}+\int_{0}^{2 \pi}\left(\frac{8 \pi^{3} S d_{4}}{L^{3}}\right)\left(\partial_{\alpha}^{3}\left(\theta-\theta^{\prime}\right)\right) H \partial_{\alpha}^{2}\left(\gamma-\gamma^{\prime}\right) d \alpha .
$$

We now add (113), (115), and (116), arriving at the following:

$$
\begin{aligned}
& \frac{d\left(Z_{1}+Z_{2}+Z_{3}+Z_{4}\right)}{d t} \leq c E_{d} \\
& \quad+\int_{0}^{2 \pi}\left(-\frac{4 \pi^{3} S d_{2} \theta_{\alpha}^{2}}{L^{3}}+\frac{2 \pi S d_{2} \bar{c}_{1}}{L}-\frac{2 \pi^{2} d_{3}}{L^{2}}+\frac{8 \pi^{3} S d_{4}}{L^{3}}\right)\left(\partial_{\alpha}^{3}\left(\theta-\theta^{\prime}\right)\right) H \partial_{\alpha}^{2}\left(\gamma-\gamma^{\prime}\right) d \alpha .
\end{aligned}
$$

The choice of $d_{3}$ and $d_{4}$ depends on the sign of $\bar{c}_{1}$; this is exactly the same as occured when choosing $c_{3}$ and $c_{4}$ in the proof of Theorem 10. Indeed, our choices of $d_{3}$ and $d_{4}$ are almost identical:

$$
\begin{gathered}
d_{3}=\frac{L S d_{2}\left(\bar{c}_{1}\right)^{+}}{\pi}+\frac{4 \pi S}{L}, \\
d_{4}=\frac{L^{2} d_{2}\left(\bar{c}_{1}\right)^{-}}{4 \pi^{2}}+\frac{d_{2}}{2} \theta_{\alpha}^{2}+1 .
\end{gathered}
$$

With these choices, then, the integral on the right-hand side of (117) vanishes. Combining (117) with (107), we conclude

$$
\frac{d E_{d}}{d t} \leq c E_{d}
$$

As discussed at the beginning of the proof, this completes the proof. 


\section{Conclusion}

We have presented a well-posedness theory for the initial value problem describing the evolution of hydroelastic waves in two dimensions. Our model assumes a thin, massless elastic sheet interacts with an inviscid, irrotational flow. The elastic model accounts for membrane bending stresses and surface tension. We prove short time well-posedness in Sobolev spaces. More precisely, given periodic initial data $\theta(\cdot,) \in H^{s}$ and $\gamma(\cdot, 0) \in H^{s-3 / 2}$ for $s$ large enough so that our estimates hold, there is a nonzero time in which the solution exists, is unique, has the same regularity as the initial conditions, and depends continuously on the data. The proof is based on energy estimates, and makes use of an arclength-angle representation of the interface and a small scale decomposition first introduced for computational reasons in [25].

In future work, we expect to treat the three-dimensional problem, as well as the problem with mass (in either two or three dimensions). To replace the arclength parameterization, we expect to use a generalized isothermal parameterization as discussed in [8], [9].

\section{Acknowledgments}

This work was supported by the NSF under Grant Nos. DMS-1009105, DMS-1016406, and DMS1412789 (MS) and DMS-1016267 (DMA).

\section{Appendix: Pressure jump at the interface}

The elastic interface deforms due to the pressure exterted on it by the fluids. In this section, the relation (19) for the pressure jump at the interface is derived. The approach follows [12].

We imagine the $1 \mathrm{D}$ interface $(x(\alpha, t), y(\alpha, t))$ to be the trace in the $x-y$ plane of a $2 \mathrm{D}$ elastic sheet or plate with no variation in the $z$-direction. Suppressing the dependence on time, let $\mathbf{F}(s)$ be the resultant internal force and $\mathbf{M}(s)$ the moment of internal force (both per unit length in the $z$-direction) on a cross section of the sheet, which is assumed to have thickness $h$. The resultant external force on the sheet is the jump in pressure at the interface, $[p]=\left.\left(p_{1}-p_{2}\right)\right|_{S}$. The equations of mechanical equilibrium for the deformed sheet or plate are [22], [30]

$$
\frac{d \mathbf{F}}{d s}=-[p] \hat{\mathbf{n}}, \quad \frac{d \mathbf{M}}{d s}=\mathbf{F} \times \hat{\mathbf{t}} .
$$

Decompose $\mathbf{F}(s)$ into tangential and normal components as $\mathbf{F}(s)=T(s) \hat{\mathbf{t}}+N(s) \hat{\mathbf{n}}$, and note that $\mathbf{M}(s)$ is in the $z$-direction so we can write $\mathbf{M}(s)=M(s) \hat{\mathbf{k}}$. Substitute these relations into (118), take the derivative with respect to $s$ and use the Frenet formulae to obtain

$$
\begin{aligned}
\left(T^{\prime}-N \kappa\right) \hat{\mathbf{t}}+\left(N^{\prime}+\kappa T\right) \hat{\mathbf{n}} & =-[p] \hat{\mathbf{n}}, \\
M^{\prime} & =-N,
\end{aligned}
$$

where the prime denotes derivative with respect to $s$. Equating normal and tangential components in (119), we obtain the system of equations

$$
\begin{array}{r}
T^{\prime}-N \kappa=0, \\
N^{\prime}+\kappa T=-[p], \\
M^{\prime}+N=0 .
\end{array}
$$


We assume a linear constitutive relationship for the elastic moment [34]

$$
M=E_{B} \kappa
$$

where $E_{B}$ is the bending modulus. Thin shell theory provides the relation $E_{B}=E h^{3} /\left[12\left(1-\nu^{2}\right)\right]$, where $E$ is Young's modulus, $\nu$ is Poisson's ratio and $h$ is the plate thickness. Insert (121) into the third equation of (120) to eliminate $M$ and $N$ and integrate once with respect to arclength to obtain the relation for the pressure jump

$$
[p]=E_{b} \kappa^{\prime \prime}+\frac{E_{B}}{2} \kappa^{3}-c_{1}(t) \kappa .
$$

where $c_{1}(t)$ is a constant (in space) of integration. The first two terms on the right hand side of (19) represent the internal bending stress of the elastic sheet, and the thrid term is surface tension. This result is consistent with the hydroelastic model of Plotnikov and Toland [32].

\section{References}

[1] S. Alben and M.J. Shelley. Flapping states of a flag in an inviscid fluid: bistability and the transition to chaos. Phys. Rev. Lett., 100(7):074301, 2008.

[2] D.M. Ambrose. Well-posedness of vortex sheets with surface tension. ProQuest LLC, Ann Arbor, MI, 2002. Thesis (Ph.D.)-Duke University.

[3] D.M. Ambrose. Well-posedness of vortex sheets with surface tension. SIAM J. Math. Anal., 35(1):211-244 (electronic), 2003.

[4] D.M. Ambrose. Well-posedness of two-phase Hele-Shaw flow without surface tension. European J. Appl. Math., 15(5):597-607, 2004.

[5] D.M. Ambrose. The zero surface tension limit of two-dimensional interfacial Darcy flow. $J$. Math. Fluid Mech., 2013. Accepted.

[6] D.M. Ambrose. The zero surface tension limit of two-dimensional interfacial darcy flow. $J$. Math. Fluid Mech., 16(1):105-143, 2014.

[7] D.M. Ambrose and N. Masmoudi. The zero surface tension limit of two-dimensional water waves. Comm. Pure Appl. Math., 58(10):1287-1315, 2005.

[8] D.M. Ambrose and N. Masmoudi. Well-posedness of 3d vortex sheets with surface tension. Comm. Math. Sci., 5(2):391-430, 2007.

[9] D.M. Ambrose, M. Siegel, and S. Tlupova. A small-scale decomposition for 3D boundary integral computations with surface tension. J. Comp. Phys., 247(0):168 - 191, 2013.

[10] G.R. Baker, D.I. Meiron, and S.A. Orszag. Generalized vortex methods for free-surface flow problems. J. Fluid Mech., 123:477-501, 1982.

[11] J.T. Beale, T.Y. Hou, and J.S. Lowengrub. Growth rates for the linearized motion of fluid interfaces away from equilibrium. Comm. Pure Appl. Math., 46(9):1269-1301, 1993. 
[12] M.G. Blyth, E.I. Părău, and J.-M. Vanden-Broeck. Hydroelastic waves on fluid sheets. J. Fluid Mech., 689:541-551, 2011.

[13] A. Chambolle, B. Desjardins, M.J. Esteban, and C. Grandmont. Existence of weak solutions for the unsteady interaction of a viscous fluid with an elastic plate. J. Math. Fluid Mech., $7(3): 368-404,2005$.

[14] C.H.A. Cheng, D. Coutand, and S. Shkoller. Navier-Stokes equations interacting with a nonlinear elastic biofluid shell. SIAM J. Math. Anal., 39(3):742-800, 2007.

[15] C.H.A. Cheng and S. Shkoller. The interaction of the 3d navier-stokes equations with a moving nonlinear koiter elastic shell. SIAM J. Math. Anal., 42(3):1094-1155, 2010.

[16] H. Christianson, V.M. Hur, and G. Staffilani. Strichartz estimates for the water-wave problem with surface tension. Comm. Partial Differential Equations, 35(12):2195-2252, 2010.

[17] A. Córdoba, D. Córdoba, and F. Gancedo. Interface evolution: water waves in 2-D. Adv. Math., 223(1):120-173, 2010.

[18] A. Cordoba, D. Cordoba, and F. Gancedo. Interface evolution: water waves in 2-D. Adv. Math, 223(7):120-173, 2010.

[19] A. Córdoba, D. Córdoba, and F. Gancedo. Interface evolution: the Hele-Shaw and Muskat problems. Ann. of Math. (2), 173(1):477-542, 2011.

[20] D. Coutand and S. Shkoller. Motion of an elastic solid inside an incompressible viscous fluid. Arch. Rat. Mech. Anal., 176(1):25-102, 2005.

[21] W.-P. Düll. Validity of the Korteweg-de Vries approximation for the two-dimensional water wave problem in the arc length formulation. Comm. Pure Appl. Math., 65(3):381-429, 2012.

[22] J.E. Flaherty, J. B. Keller, and S.I. Rubinow. Post buckling behavior of elastic tubes and rings with opposite sides in contact. SIAM J. Appl. Math., 23(4):446-455, 1972.

[23] Y. Guo, C. Hallstrom, and D. Spirn. Dynamics near unstable, interfacial fluids. Comm. Math. Phys., 270(3):635-689, 2007.

[24] H. Helson. Harmonic analysis. Addison-Wesley Publishing Company Advanced Book Program, Reading, MA, 1983.

[25] T.Y. Hou, J.S. Lowengrub, and M.J. Shelley. Removing the stiffness from interfacial flows with surface tension. J. Comput. Phys., 114(2):312-338, 1994.

[26] T.Y. Hou, J.S. Lowengrub, and M.J. Shelley. The long-time motion of vortex sheets with surface tension. Phys. Fluids, 9(7):1933-1954, 1997.

[27] D. Hu, P. Zhang, and W. E. Continuum theory of a moving membrane. Physical Review E, 75(4):041605, 2007.

[28] L.-B. Jia, F. Li, X.-Z. Yin, and X.-Y. Yin. Coupling modes between two flapping filaments. J. Fluid Mech., 581:199-220, 2007. 
[29] A. Korobkin, E.I. Părău, and J.-M. Vanden-Broeck. The mathematical challenges and modelling of hydroelasticity. Phil. Trans. Roy. Soc. A: Math. Phys, Eng. Sci., 369(1947):2803-2812, 2011.

[30] L.D. Landau and E.M. Lifshitz. Elasticity theory. 1975.

[31] A.J. Majda and A.L. Bertozzi. Vorticity and incompressible flow, volume 27 of Cambridge Texts in Applied Mathematics. Cambridge University Press, Cambridge, 2002.

[32] P.I. Plotnikov and J.F. Toland. Modelling nonlinear hydroelastic waves. Phil. Trans. Roy. Soc. A: Math. Phys, Eng. Sci., 369(1947):2942-2956, 2011.

[33] P.I. Plotnikov and J.F. Toland. Strain-gradient theory of hydroelastic travelling waves and young measures of their singular limits. Calc. Var. PDE, 44(1-2):153-197, 2012.

[34] C. Pozrikidis. Buckling and collapse of open and closed cylindrical shells. J. Eng. Math., 42(2):157-180, 2002.

[35] V.A. Squire. Synergies between VLFS hydroelasticity and sea ice research. Int. J. Offshore Polar Eng., 18(4):241-253, 2008.

[36] V.A. Squire, J.P. Dugan, P. Wadhams, P.J. Rottier, and A.K. Liu. Of ocean waves and sea ice. Ann. Rev. of Fluid Mech., 27(1):115-168, 1995.

[37] M.E. Taylor. Partial differential equations. III, volume 117 of Applied Mathematical Sciences. Springer-Verlag, New York, 1997. Nonlinear equations, Corrected reprint of the 1996 original.

[38] J.F. Toland. Heavy hydroelastic travelling waves. Proc. Roy. Soc. A: Math. Phys, Eng. Sci., 463(2085):2371-2397, 2007.

[39] J.F. Toland. Steady periodic hydroelastic waves. Arch. Rat. Mech. Anal., 189(2):325-362, 2008.

[40] W. Wang, P. Zhang, and Z. Zhang. Well-posedness of hydrodynamics on the moving elastic surface. Arch. Rat. Mech. Anal., 206(3):953-995, 2012.

[41] S. Wu. Well-posedness in Sobolev spaces of the full water wave problem in 2-D. Invent. Math., 130(1):39-72, 1997.

[42] J. Ye and S. Tanveer. Global existence for a translating near-circular Hele-Shaw bubble with surface tension. SIAM J. Math. Anal., 43(1):457-506, 2011.

[43] J. Ye and S. Tanveer. Global solutions for a two-phase Hele-Shaw bubble for a near-circular initial shape. Complex Var. Elliptic Equ., 57(1):23-61, 2012.

[44] E. Zeidler. Nonlinear functional analysis and its applications. I. Springer-Verlag, New York, 1986. Fixed-point theorems, Translated from the German by Peter R. Wadsack. 